\title{
NAFLD, cardiovascular and cardiac diseases: factors influencing risk, prediction, and treatment
}

Giovanni Targher, $\mathrm{MD}^{1^{*}}$, Kathleen E. Corey, MD, MPH, MMSc${ }^{2 *}$, Christopher D. Byrne, MB BCh, $\mathrm{PhD}^{3,4^{*}}$

${ }^{*}$ All three authors contributed equally to the manuscript

${ }^{1}$ Section of Endocrinology, Diabetes and Metabolism, Department of Medicine, University and Azienda Ospedaliera Universitaria Integrata of Verona, Verona, Italy

${ }^{2}$ Liver Center, Division of Gastroenterology, Department of Medicine, Massachusetts General Hospital and Harvard Medical School, Boston, MA, USA

${ }^{3}$ Nutrition and Metabolism, Faculty of Medicine, University of Southampton, UK ${ }^{4}$ Southampton National Institute for Health Research Biomedical Research Centre, University Hospital Southampton, Southampton General Hospital, Tremona Road, Southampton, UK

Word count: 246 abstract; 7800 text (excluding title page, abstract, references, tables and figure legends). Figures: 4, Tables: 1

\author{
Address for correspondence: \\ Prof. Giovanni Targher, MD \\ Section of Endocrinology, Diabetes and Metabolism \\ University and Azienda Ospedaliera Universitaria Integrata \\ Piazzale Stefani, 1 \\ 37126 Verona, Italy \\ Phone: 0039/045-8123748 \\ E-mail: giovanni.targher@univr.it
}

\section{LIST OF ABBREVIATIONS}

NAFLD, nonalcoholic fatty liver disease; NASH, nonalcoholic steatohepatitis; CVD, cardiovascular disease; T2DM, type 2 diabetes mellitus; MetS, metabolic syndrome; AF, atrial fibrillation; PNPLA3, patatin-like phospholipase domain-containing protein 3; SNP, single nucleotide polymorphism; TM6SF2, trans-membrane 6 superfamily 2; LDL, low-density lipoprotein; sd-LDL, small dense LDL; HDL-C, high density lipoprotein cholesterol; VLDL, very low density lipoprotein; HR, hazard ratio; OR, odds ratio; $95 \% \mathrm{Cl}, 95 \%$ confidence interval; LPS, lipopolysaccharide; TMA, trimethylamine; TMAO, trimethylamine oxide; FRS, Framingham risk score; ASCVD, atherosclerotic cardiovascular disease score; SGLT2, sodium-glucose cotransporter-2; GLP-1 RA, glucagon-like peptide-1 receptor agonist; HF, heart failure. 
Background/Aim: Non-alcoholic fatty liver disease (NAFLD), affecting up to $\sim 30 \%$ of the world adult population, causes considerable liver-related and extra-hepatic morbidity and mortality. Strong evidence indicates that NAFLD (especially in its more severe forms) is associated with a higher risk of all-cause mortality, and the predominant cause of mortality in this patient population is cardiovascular disease (CVD). The aim of this narrative review is to discuss the strong association between NAFLD and increased risk of cardiovascular, cardiac and arrhythmic complications. We also discuss the putative mechanisms that link NAFLD to CVD and other cardiac/arrhythmic complications; and briefly summarize the CVD risk prediction/stratification and management of the increased risk for CVD observed in patients with NAFLD.

Results: NAFLD is associated with an increased risk of CVD events and other cardiac complications (left ventricular hypertrophy, valvular calcification and certain arrhythmias), independently of common CVD risk factors. The magnitude of risk of CVD and other cardiac/arrhythmic complications parallels the severity of NAFLD (especially the severity of liver fibrosis). There are likely multiple underlying mechanisms by which NAFLD may increase the risk of CVD and cardiac/arrhythmic complications. Indeed, NAFLD exacerbates hepatic and systemic insulin resistance, promotes atherogenic dyslipidaemia, induces hypertension and causes the synthesis of pro-atherogenic, pro-coagulant and pro-inflammatory mediators that may contribute to the development of CVD and other cardiac/arrhythmic complications.

Conclusions: A careful assessment of CVD risk is mandatory in patients with NAFLD for the primary prevention of CVD, together with pharmacological treatment of coexisting CVD risk factors.

Keywords: Nonalcoholic fatty liver disease, NAFLD, CVD, arrhythmias, heart disease 


\section{INTRODUCTION}

Non-alcoholic fatty liver disease (NAFLD) is a public health problem worldwide (affecting up to a third of adults in Western countries), and its prevalence is expected to further increase in the near future at a similar rate as the obesity and type 2 diabetes mellitus (T2DM) epidemics [1, 2].

The clinical and economic burden of this common liver disease is not only due to its liver-related complications (non-alcoholic steatohepatitis [NASH], cirrhosis or hepatocellular carcinoma), but also due to an increased risk of cardiovascular disease (CVD) and other extra-hepatic diseases (e.g. T2DM, chronic kidney disease or some extra-hepatic cancers) that impact health outcomes and expenditures [2-5].

It is known that CVD is the leading cause of mortality amongst patients with NAFLD (accounting for $\sim 40 \%$ of the total deaths), followed by extra-hepatic cancers and liverrelated complications [2-4]. Over the last decade, it has become evident that NAFLD is a 'multi-system' disease [6] that not only increases the risk of developing CVD, T2DM or chronic kidney disease [2,7], but also results in other cardiac and arrhythmic complications (Figure 1) [8].

This narrative review focuses on the strong association between NAFLD and increased risk of CVD and other cardiac/arrhythmic complications; discusses the potential underlying mechanisms by which NAFLD contributes to the development of these complications; and summarizes the CVD risk prediction/stratification and management of the CVD risk associated with NAFLD.

\section{RISK OF CVD AND OTHER CARDIAC AND ARRHYTHMIC COMPLICATIONS IN NAFLD}

\subsection{Risk of CVD}

As previously reported, CVD is the leading cause of death in NAFLD.[2-4] Using mortality data from the National Vital Statistics System multiple-cause mortality data in the United States, Paik et al. recently confirmed that CVD was one of the most important causes of death amongst NAFLD patients [9].

Convincing evidence associates NAFLD with an increased prevalence and incidence of CVD even after adjustment for common CVD risk factors [2, 4, 10]. Table 1 shows the quantification of the excess of CVD risk in patients with NAFLD (i.e., subclinical atherosclerosis or adverse CVD outcomes). In a recent meta-analysis of 26 crosssectional studies involving 85,395 individuals, Zhou et al. [11] reported that NAFLD was associated with markers of subclinical atherosclerosis (pooled random-effects odds ratio [OR] 1.60; 95\% confidence interval [Cl] 1.45-1.78). Subgroup analyses revealed that NAFLD was associated with greater carotid artery intima-media thickness/plaques, increased arterial stiffness, increased coronary artery calcification and endothelial dysfunction with ORs of $1.74(95 \% \mathrm{Cl} 1.47-2.06), 1.56(95 \% \mathrm{Cl} 1.24-$ $1.96), 1.40$ (95\% Cl 1.22-1.60), and 3.73 (95\% Cl 0.99-14.09), respectively [11]. In a meta-analysis of 16 longitudinal studies (involving $\sim 34,000$ middle-aged participants followed for a median follow-up of 6.9 years), Targher et al. showed that NAFLD was associated with a higher risk of fatal and/or non-fatal CVD events (random-effects OR $1.64,95 \% \mathrm{Cl} 1.26-2.13$ ) [12]. In addition, this risk increased further with greater severity of NAFLD (random-effects OR 2.58; 95\% Cl 1.78-3.75), and remained significant in those studies where analysis was adjusted for conventional CVD risk factors [12]. 
Recently, other studies reported an independent association between NAFLD and risk of incident acute myocardial infarction, even in primary care populations [13, 14]. However, this finding was questioned by the recent results of a matched cohort study [15]. Using electronic medical records from four European primary healthcare databases, the authors failed to find an independent association between recorded diagnoses of NAFLD and risk of incident acute myocardial infarction or ischemic stroke [15]. However, it should be noted that the prevalence of NAFLD in this electronic database was $\sim 15$ fold lower than that reported in the general population when imaging techniques are used for the diagnosis of NAFLD, highlighting the potential for misclassification bias within the 'control' group [10].

Interestingly, retrospective cohort studies performed in Asian adults without preexisting CVD also reported that there was a strong association between NAFLD and the risk of progression of subclinical carotid or coronary atherosclerosis, and, most importantly, that improvement or resolution of NAFLD (on ultrasonography) was associated with a lower risk of carotid atherosclerosis development $[10,16,17]$.

Collectively, therefore, although further cohort studies of patients with histologically confirmed NAFLD are required, a number of studies with relatively long follow-ups have confirmed the results of our aforementioned meta-analysis [12]. Furthermore, evidence is accumulating that the risk of incident CVD events parallels the severity of NAFLD histology, and the stage of fibrosis is the strongest histologic predictor of adverse liverrelated and CVD outcomes in NAFLD [18-20]. Recently, Henson et al. [21] examined the factors associated with incident CVD events in a cohort of adults with biopsy-proven NAFLD and without pre-existing CVD. Using a competing risks analysis, the authors showed that advanced liver fibrosis was a significant predictor of incident CVD events during a median follow-up period of 5.2 years, and this persisted on multivariable analyses even after considering relevant covariates, including CVD risk scores, which were not independent predictors. Notably, other histologic features, including NASH, were not associated with risk of CVD events [21]. As stated by the authors [21], the findings of this study should be considered when evaluating patients with NAFLD for primary prevention of CVD, and further investigation into the link between advanced liver fibrosis and CVD risk is needed.

\subsection{Risk of cardiac arrhythmias and conduction defects}

In the past few years, the link between NAFLD and the increased risk of arrhythmias (mainly permanent atrial fibrillation [AF]) has gained great scientific interest [8][10]. As also shown in Table 1, in a meta-analysis of 9 cohort studies (involving $\sim 365,000$ individuals), Mantovani et al. [22] showed that NAFLD doubled the risk of prevalent AF (random-effects OR 2.07, 95\% $\mathrm{Cl} 1.38-3.10$ ), independent of common AF risk factors. This risk was particularly high amongst patients with T2DM. In addition, meta-analysis of data from longitudinal studies also showed that NAFLD was associated with increased risk of incident AF, especially among those with established T2DM (randomeffects hazard ratio [HR] 4.96, 95\%Cl 1.42-17.3) [22]. A larger meta-analysis of 6 cohort studies (involving $\sim 615,000$ individuals with and without T2DM) recently confirmed the existence of an independent association between NAFLD and risk of incident AF (random-effects HR 1.19, 95\% Cl 1.04-1.31) [23]. In a retrospective cohort of $\sim 75,000$ South Korean individuals with NAFLD without a history of liver or cardiovascular diseases, Park et al. [24] reported that NAFLD patients with increased advanced fibrosis scores had a higher risk of prevalent AF even after adjusting for multiple CVD risk factors (adjusted-OR 2.23, 95\% Cl 1.74-2.91). 
Increasing evidence suggests that the presence and severity of NAFLD is associated with a higher risk for QTC interval prolongation (on standard electrocardiogram) that predisposes to increased risk of both ventricular tachyarrhythmias and sudden cardiac death [8]. In particular, in a cohort of $\sim 30,000$ Taiwanese individuals, Hung et al. found a significant, graded relationship between the ultrasonographic severity of NAFLD and risk of QTc interval prolongation even after adjusting for common CVD risk factors [25]. Similarly, Mantovani et al. found an association between NAFLD and risk of ventricular tachyarrhythmias in a cohort of T2DM patients referred for clinically indicated 24-hour Holter monitoring. This association was independent of age, sex, obesity, hypertension, smoking, co-morbidities, medication use and left ventricular (LV) ejection fraction [26].

Finally, preliminary evidence suggests the existence of an association between NAFLD and certain cardiac conduction defects, which are risk factors of cardiac mortality [8]. In a hospital-based case-control study of 700 patients without known cardiovascular and liver diseases, Mangi et al. [27] reported a significant association between NAFLD and presence of certain types of cardiac conduction defects (on standard electrocardiogram). Similarly, Mantovani et al. [28] found that NAFLD was associated with a threefold increased risk of cardiac conduction defects (mainly persistent firstdegree atrio-ventricular block, right bundle branch block or left anterior hemi-block) in a hospital-based cohort of 751 elderly patients with T2DM.

Taken together, the results of these studies support the existence of a strong relationship between NAFLD and cardiac arrhythmias, such as permanent AF, QTC interval prolongation and ventricular tachyarrhythmias. Although further research is needed, we believe that these findings are clinically relevant, as they could partly explain the increased risk of cardiac death observed in patients with NAFLD.

\subsection{Risk of functional and structural myocardial abnormalities}

Convincing evidence now substantiates a strong association between NAFLD and risk of LV diastolic dysfunction and hypertrophy both in children and in adults, with or without co-existing features of the metabolic syndrome (MetS) [8] [10].

As reported in Table 1, in a meta-analysis of 12 cross-sectional studies, Wijarnpreecha et al. [29] reported an independent association between NAFLD and LV diastolic dysfunction with a pooled random-effects OR of $2.02(95 \% \mathrm{Cl} 1.47-2.79)$. This finding was recently confirmed among the Framingham Heart Study participants, who underwent echocardiography and standardized computed tomography measures of liver fat [30]. Additionally, in a cohort study of 3,300 South Korean individuals, Chung et al. [31] found that the NAFLD-associated risk of LV diastolic dysfunction incrementally increased according to the presence of advanced liver fibrosis (estimated by non-invasive fibrosis scores). In a sample of 308 South Korean patients, Lee et al. [32] also showed that hepatic steatosis and fibrosis (as assessed by vibrationcontrolled transient elastography) were independently associated with LV diastolic dysfunction. This association was linked to altered myocardial glucose uptake evaluated by 18-fluoro-deoxyglucose-positron emission tomography.

Some smaller studies using liver biopsy to diagnose NAFLD also reported a graded, positive association between myocardial abnormalities (i.e., LV diastolic dysfunction, greater cardiac mass or larger left atrial volume) and the severity of NAFLD histology [33-35]. 


\subsection{Risk of valvular calcification}

Aortic valve sclerosis and mitral annular calcification are two common findings in elderly individuals that are associated with adverse CVD outcomes [8, 36, 37].

As reported in Table 1, Di Minno et al. [38] reported a significant association between NAFLD and increased prevalence of aortic valve sclerosis (random-effects OR 2.28, $95 \% \mathrm{Cl} 1.21-4.28$ ) in a meta-analysis of three cross-sectional studies enrolling a total of 2,639 middle-aged individuals. More recently, Mantovani et al. [39] found that ultrasound-detected NAFLD was associated with a $\sim 3.5$ fold higher risk of both aortic valve sclerosis and mitral annular calcification in a hospital-based study of T2DM patients with no history of heart failure, moderate-to-severe heart valve diseases or known liver diseases. This association remained significant after adjusting for traditional CVD risk factors, diabetes-related variables, medication use and some relevant echocardiographic variables [39].

\section{PUTATIVE MECHANISMS}

NAFLD and T2DM frequently co-exist and share CVD risk factors in common, for example NAFLD and T2DM are both strongly associated with factors linked to the MetS, including increased abdominal adiposity.[40] Whereas the increase in risk of T2DM with NAFLD is independent of potential confounders [41], it is more difficult to prove that the association between NAFLD and CVD is independent of cardiometabolic risk factors that are shared with T2DM. Furthermore, with both NAFLD and T2DM, a "vicious spiral" of worsening disease also exists, with the presence of either disease worsening the development and progression of the other disease. For example, there is now unequivocal evidence that NAFLD is a risk factor for T2DM [41], and T2DM increases risk for CVD and advanced liver disease (e.g., NASH, cirrhosis or hepatocellular carcinoma) [42-47]. Additionally, recent evidence (discussed below) suggests that with the development of advanced liver disease in NAFLD, there is a further increase in risk of CVD events, compared to the presence of liver fat alone. Figure 2 illustrates schematically, the "vicious spiral" of worsening disease that occurs when NAFLD co-exists with T2DM, to increase the risk of CVD and cardiac diseases (panel A) and summarizes some of the potential CVD and cardiac risk factors in NAFLD (panel B).

Although as discussed above, there is evidence that NAFLD may be an independent risk factor for CVD [12], the evidence also suggests that NAFLD is a stronger risk factor for T2DM [41], and with the development of T2DM, the presence of diabetes is an additional established CVD risk factor. With NAFLD, fatty liver alone increases the risk of incident T2DM at least two fold [41]. However, when fatty liver is combined with obesity and insulin resistance (that also commonly occur with NAFLD), there is an additive effect of all three risk factors, further markedly increasing the risk of T2DM by $\sim 12$ fold [48]. Until recently, it had been thought by some that the coexistence of T2DM confounded the association between NAFLD and CVD. However, Wild et al. showed that this was not the case, and confirmed that NAFLD was an independent risk factor for CVD, even in people with established T2DM [43]. These investigators used data from the diabetes registry in Scotland between 2004 and 2013. Patients had one or more hospital admission records, and linked hospital, cancer, and death records and liver disease and outcomes, were identified using ICD-9 and ICD-10 codes. For 
NAFLD, the adjusted HRs, compared with the group with no record of liver disease, were $1.70(95 \% \mathrm{Cl} 1.52,1.90)$ for CVD and $1.60(95 \% \mathrm{Cl} 1.40,1.83)$ for all-cause mortality, respectively [43]. Moreover, in support of these findings that T2DM does not confound the association between NAFLD and risk of CVD events, a recent metaanalysis confirmed that NAFLD was associated with a higher risk of fatal and/or nonfatal CVD events in T2DM patients [49].

T2DM is not only associated with increased adiposity but also with changes in lipoprotein metabolism; changes in intestinal function and alterations in the intestinal microbiome (dysbiosis); and these factors may exert an adverse effect on the risk of CVD and cardiac diseases in NAFLD. Figure 3 illustrates schematically the development of atherogenic dyslipidaemia in NAFLD and its relationship with MetS. The figure also highlights the role of intestinal factors and adipose tissue function to deliver long-chains fatty acids (LCFAs) as substrates and stimuli for various liver lipid species, which in turn affect hepatic inflammation, insulin resistance, oxidative stress and lipid globule formation in NAFLD.

\subsection{Lipoprotein metabolism, MetS and genetic modifiers of CVD risk}

NAFLD is frequently a cause of atherogenic dyslipidaemia, which is characterized by increased levels of very-low density lipoprotein (VLDL), small dense LDL (sd-LDL), lower levels of high density lipoprotein cholesterol (HDL-C) together with postprandial lipemia and HDL dysfunction [50,51]. Not only has atherogenic dyslipidaemia been observed in adults but recently this dyslipidaemia has also been noted in children with NAFLD [52]. In this study, nuclear magnetic resonance lipoprotein profile, including lipoprotein particle sizes, apolipoproteins and the lipoprotein-insulin resistance index, were measured in 76 children at the time of liver biopsy. Children with NASH had higher ratios of apolipoprotein $B$ to apolipoprotein $\mathrm{Al}$ (ApoB/ApoAI) and higher lipoproteininsulin resistance index than children with non-NASH. Severity of hepatocyte ballooning was also associated with higher ApoB/ApoAl ratios, while HDL size was inversely associated with hepatic fat accumulation [52]. Thus, these interesting data in children, who are unlikely to have other comorbidities (such as T2DM that was excluded), suggest that it is the liver disease that is responsible for the dyslipidaemia and not co-existing diabetes. Thus, atherogenic dyslipidaemia may be, at least in part, responsible for the increased risk of CVD in NAFLD, since it is well accepted that this dyslipidaemia increases CVD risk [51, 53-55]. As mentioned above, increased sd-LDL particles are also a feature of this dyslipidaemia, and increased sd-LDL levels are associated with NAFLD and MetS [56-59]. Thus, it is plausible that the increased CVD associated with more severe liver disease in NAFLD may, at least in part, be linked to increased levels of sd-LDL, since higher levels of this lipoprotein have been reported in patients with NASH than in those with fatty liver alone [60].

When considering the association between NAFLD and CVD, no studies to date have satisfactorily taken account of the potential modifying influence of common genetic polymorphisms (SNP) in NAFLD and the influence that these SNPs may have on NAFLD-related CVD risk. The two most frequently studied SNPs in NAFLD, i.e., the patatin-like phospholipase domain-containing protein 3 (PNPLA3) rs738409 and the trans-membrane 6 superfamily 2 (TM6SF2) rs58542926 genetic variants, are known to be associated with a higher risk of NASH and yet lower levels of serum triglycerides [61-63]. In a cohort of 300 Finnish individuals genotyped for both the E167K (rs58542926) variant in TM6SF2 and the I148M (rs738409) variant in the PNPLA3 gene in whom liver fat content was measured by magnetic resonance spectroscopy, circulating lipids were analyzed by liquid chromatography-mass chromatography [61]. 
The authors compared the plasma lipidome between three groups of NAFLD patients with similar levels of liver fat: (i) carriers of the E167K but not of the I148M variant in PNPLA3 (the 'TM6SF2 NAFLD' group), (ii) carriers of the I148M but not of the E167K variant (the 'PNPLA3 NAFLD' group), and (iii) non-carriers of either risk allele. In this analysis, the authors showed that, despite similar liver fat levels between these three groups, both the 'TM6SF2 NAFLD' and 'PNPLA3 NAFLD' groups had lower levels of serum triglycerides and most common triacylglycerols than non-carriers of either risk allele. Since fasting triglyceride levels are predominantly hepatic-derived VLDL particles, and lower levels of serum triglycerides are associated with higher levels of HDL-C and lower levels of sd-LDL (i.e., atherogenic dyslipidaemia as discussed above), both the 'TM6SF2 NAFLD' and 'PNPLA3 NAFLD' groups had a less atherogenic plasma lipid profile that is associated with lower CVD risk, despite both risk alleles being associated with more severe liver disease in NAFLD.

In support of the notion that these PNPLA3 or TM6SF2 risk alleles have the potential to modify the CVD risk in NAFLD despite being a potential cause for more severe liver disease, a recent population-based exome-focused genotyping array study tested associations between PNPLA3 or TM6SF2 risk alleles and CVD risk in the general population using the UK BioBank cohort [64]. The data were obtained from $>300,000$ participants and replicated in $>280,000$ participants in the cohort. The results of this analysis identified 444 variants in 250 genetic loci that were associated with plasma lipid profile. As discussed above, both PNPLA3 and TM6SF2 risk alleles were associated with lower levels of serum triglycerides, higher levels of liver fat content and a higher risk of T2DM; also providing indirect evidence that increased liver fat was associated with higher risk of T2DM. Interestingly, in this study both PNPLA3 and TM6SF2 risk alleles were associated with lower risk of CVD [64]. Thus, these data provide further insight into the association between NAFLD and CVD risk as patients carrying these two genotypes represent a subgroup of NAFLD patients who are at lower CVD risk, because they have lower levels of hepatic-derived lipoproteins.

Previously, in a meta-analysis of studies investigating the relationship between NAFLD and risk of CVD events, we have shown that NAFLD is independently associated with increased risk of CVD [12]. The data discussed above [64], showing that PNPLA3 or TM6SF2 risk alleles are associated with a lower risk of CVD (despite increased liver fat), suggests that the lower levels of hepatic-derived lipoproteins (VLDL, IDL and LDL) with these risk alleles may be the key mediator of the decreased risk of CVD in NAFLD patients carrying these risk alleles, compared to the increased risk of CVD in those who don't have these risk alleles. The mechanism by which PNPLA3 I148M lowers hepatic-derived lipoproteins has recently been studied in humans [65]. Although in this small study comparing 12 subjects with PNPLA3 M148M with 14 subjects with PNPLA3 I148I, there was no difference between these two groups in total VLDL levels, subjects with PNPLA3 M148M had increased polyunsaturated triacylglycerols, and phosphatidylcholines were decreased in liver. These data led the authors to conclude that PNPLA3-148M is a loss-of-function allele that remodels intra-hepatic triglycerides in a polyunsaturated direction by impairing hydrolysis/transacylation of polyunsaturated fatty acids from diacylglycerols to feed phosphatidylcholine synthesis [65]. Additionally, the mechanism by which the TM6SF2E167K variant lowers VLDL levels has also recently been studied, using 3D hepatic spheroids derived from primary human hepatocytes [66]. These data showed that the TM6SF2E167K variant had

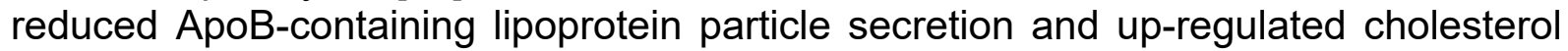
and fatty acid biosynthesis. Taken together, these in vivo and in vitro data suggests that both PNPLA3 and TM6SF2 risk alleles have effects that may influence composition and quantity of hepatic-derived triglyceride-rich lipoproteins in the circulation to attenuate risk of CVD. 
Another susceptibility gene for NAFLD is the GCKR (encoding liver-specific glucokinase regulatory protein [GKRP]) that is involved both in hepatic de novo lipogenesis [67] and in NAFLD development [68]. A recent meta-analysis reported that common variants in GCKR gene were weakly associated with the risk of CVD (OR per risk allele 1.02 [95\% Cl 1.0-1.04]) [69], and this increased CVD risk might also be due to changes in VLDL (i.e. increased) with this susceptibility gene [70].

\subsection{Inflammation, alterations in redox state, liver fibrosis and CVD}

Increased liver fat has been associated with plasma inflammatory biomarkers in the Framingham Heart Study [71]. In this community-based study, the authors used data from 2,482 middle-aged individuals, who underwent computed tomography to assess the presence of liver fat together with measurement of 14 plasma inflammatory biomarkers. Interestingly, they found that increased liver fat was associated with the following inflammatory biomarkers: high-sensitivity C-reactive protein, urinary F2isoprostanes, interleukin-6 (IL-6), intercellular adhesion molecule-1, and P-selectin. The adjustment for adiposity measures only slightly attenuated these associations [71].

Steatotic hepatocytes also release extracellular vesicles that increase endothelial inflammation [72]. In this study the vesicles from steatotic hepatocytes were characterized by altered miRNA expression profiles inducing vascular inflammation by miR-1 release and activation of NF-KB. Steatotic hepatocytes also released more extracellular vesicles compared to the extracellular vesicles released by control hepatocytes. It is plausible that steatotic hepatocyte-derived extracellular vesicles induce vascular inflammation and facilitate atherogenesis by miR-1 delivery, reduced Kruppel-like factor 4 and NF-KB activation [34]. These findings suggest a role for hepatocyte-derived extracellular vesicles affecting the vasculature, suggesting a novel mechanism underlying a link between liver disease and CVD amongst patients with NAFLD.

Hepatokine secretion also occurs with NAFLD and hepatokines may influence metabolic phenotypes by having paracrine and endocrine actions beyond the liver [7375]. Hepatokines are proteins that are secreted by hepatocytes, and hepatokines have been linked with the induction of metabolic dysfunction, insulin resistance, inflammatory pathways, and CVD [73-76]. Fetuin-A, fibroblast growth factor-21 (FGF21), retinol binding protein-4, selenoprotein- $P$, sex hormone-binding globulin, angiopoietin-related growth factor (also known as angiopoietin-related protein 6 ) and leukocyte derived chemotaxin 2 (LECT2) are considered very important hepatokines in vivo $[73,74,77]$, and recent studies have suggested that via the secretion of hepatokines the liver may directly influence whole body homeostasis [77, 78]. Preclinical studies have shown that hepatokines have important effects on glucose metabolism, lipid metabolism and body weight in animal models [76]. However, it remains less certain whether hepatokines are causally influencing CVD or cardiac diseases in patients with NAFLD. That said, a Mendelian randomisation study has suggested that serum fetuin-A concentrations are increased and might be causally involved in myocardial infarction [79]. Similarly, selenoprotein-P is associated with inflammation and in multiple regression analyses, selenoprotein- $P$ showed an independent association with carotid intima-media thickness as well as plasma Creactive protein, even after adjustment for other confounding risk factors [80]. Increased FGF-21 levels were also a significant independent risk factor for carotid intima-media thickness in regression analysis [81] and LECT2 has been shown to induce inflammatory cytokines via c-Jun N-terminal kinase (JNK) phosphorylation [82]. Nevertheless, further research is needed to determine whether modification of specific 
hepatokine concentrations, e.g. suppression of fetuin-A, selenoprotein-P or LECT2, represents an important therapeutic strategy for ameliorating CVD risk in NAFLD.

A possible link between dyslipidaemia and hepatic inflammation is also supported by recent data suggesting that proprotein convertase subtilisin/kexin type-7 gene variants are associated with the severity of liver disease in NAFLD [83]. With accumulation of lipid within hepatocytes during progression of liver disease, there is increased mitochondrial activity and oxidative stress resulting in enhanced production of reactive oxygen species, chemokines and cytokines $[84,85]$. The pathophysiology of NASH is complex, but the development of $\mathrm{NASH}$ involves several mechanisms, including increased infiltration of monocytes, T-lymphocytes and neutrophils into the liver, as well as activation and in situ expansion of the Kupffer cells or hepatic stellate cells [86]. With this process increased oxidative stress, cell death and continuous unresolved inflammation drive the activation of hepatic stellate cells, leading to increased hepatic fibrogenesis [87, 88]. As mentioned above, with progression of liver disease to advanced fibrosis, there is evidence that risk of CVD is further increased. For example, as discussed previously (Table 1), in a subgroup analysis of a meta-analysis that included 16 longitudinal studies with $\sim 34,000$ individuals, those patients with NAFLD who had evidence of more severe liver disease were also more likely to develop fatal and/or non-fatal CVD events compared to those without NAFLD [12]. Similarly, in a recent study of 1,535 patients with biopsy-proven NAFLD, the authors found that the stage of fibrosis was associated with a higher CVD risk [89]. Additionally, increased serum gamma-glutamyltransferase (GGT) levels often occur in NAFLD [90], are associated with reduced cardiorespiratory fitness in NAFLD [91], and are also associated with higher risks of all-cause and cancer mortality in the general population, even after adjusting for the presence of fatty liver [92]. GGT is an enzyme that contributes to maintaining the physiological concentrations of cytoplasmic glutathione and cellular defence against oxidative stress via cleavage of extra-cellular glutathione and increased availability of amino acids for its intra-cellular synthesis.[90] Increased GGT levels are, therefore, a marker of enhanced oxidative stress and may reflect the increased generation of free radicals and peroxidation reactions that are involved in both the plasma LDL-C oxidation and the atherosclerotic processes [90].

It is also plausible that increased cytokines and decreased adiponectin derived from expanded (dysfunctional) visceral adipose tissue may contribute either to exacerbate the severity of liver disease (via effects on lipid metabolism, glucose metabolism or insulin sensitivity) or may act directly on the vasculature. Increased plasma levels of IL-6 have also been associated with subclinical atherosclerosis in population-based studies [93]. Visceral fat also expresses much higher concentrations of IL-6, IL-1 $\beta$ and tumour necrosis factor (TNF)- $\alpha$ than the liver, and marked weight loss attenuates the expression of these cytokines [94, 95]. A meta-analysis has shown that increased levels of plasma IL-6 and CRP are associated with increased AF incidence [96]. Although there are no human studies in NAFLD that have tested the effects of specific anti-inflammatory agents that we are aware of, the effects of inhibiting a specific cytokine in patients with known vascular disease has been tested with potentially interesting results. Whether treatment to decrease IL-1 $\beta$ with canakinumab (i.e., an anti-IL-1 $\beta$ monoclonal antibody) reduces risk of major adverse CVD events was tested in the Canakinumab Anti-inflammatory Thrombosis Outcome Study that involved 10,061 patients with previous myocardial infarction and a plasma C-reactive protein level of $\geq 2 \mathrm{mg} / \mathrm{L}$ [97]. In this trial, the investigators showed that canakinumab met the pre-specified multiplicity-adjusted threshold for statistical significance for the primary endpoint and the secondary endpoint that additionally included hospitalization for unstable angina that led to urgent coronary revascularizations. However, it should be noted that compared to placebo, treatment with canakinumab was associated with a 
higher incidence of fatal infections and there was no significant difference in all-cause mortality. Thus, it remains uncertain whether specific targeted treatment of inflammation or proinflammatory mediators without also targeting proven CVD risk factors is beneficial, in conditions such as NAFLD.

\subsection{Intestinal microbiome and CVD risk}

The intestinal microbiome affects energy harvesting, inflammation and immunity and a role for the intestinal microbiota and changes in the gut microbiota (dysbiosis) have been proposed in the pathogenesis of liver fibrosis. [98]. In this study, the authors characterized the gut microbiome from stool samples in 86 patients with biopsy-proven NAFLD (72 with mild/moderate NAFLD, and 14 with advanced NAFLD). Using a set of 40 features (including 37 bacterial species), they constructed a regression model that showed excellent accuracy for distinguishing mild/moderate NAFLD from advanced fibrosis, providing intriguing preliminary evidence for a faecal-microbiome-derived metagenomic signature of advanced fibrosis in NAFLD [98].

Although patients with NAFLD have alterations in their gut microbiome (dysbiosis), changes in the microbiome are not as well defined as they are for other liver diseases, such as alcoholic liver disease. It is also possible that changes in the immune response and the metabolome induced by changes in the microbiome may have a greater impact than changes in the microbes themselves [99]. That said, one of the most commonly observed changes is an increase in Gram-negative bacteria and a decrease in Grampositive bacteria [99]. With an increase in Gram-negative bacteria and an increase in intestinal permeability, there is increased potential for endotoxin to enter the portal circulation and thereby promote an inflammatory response in the liver. Interestingly, mice given an infusion of lipopolysaccharide (LPS) developed insulin resistance, hepatic steatosis and increased adipose tissue, similar to mice fed a high fat diet [100]. Many nutritional compounds, e.g. red meat, contain a trimethylamine group (TMA), and in the liver TMA is oxidised by flavin monooxygenases (FMOs) to trimethylamine oxide (TMAO) [101]. Numerous studies have shown that plasma TMAO levels are associated with CVD, chronic kidney disease, T2DM or NAFLD [102-105]. With dysbiosis, other gastrointestinal tract-derived factors, such as LPS, aromatic acid metabolites, $p$-cresyl sulphate, indoxyl sulphate, short-chain fatty acids, incretins, and modified bile acids, may potentially contribute to exacerbate the severity of the liver disease (via effects on lipid metabolism, glucose metabolism or insulin sensitivity) or act directly on the cardiovascular system [101, 106, 107].

Although there is an unquestionable potential adverse role for dysbiosis-mediated factors both on the liver in NAFLD and on the vasculature, it is currently unclear what prebiotics, probiotics or synbiotics should be used to modify the gut microbiota. It is also poorly known which gut microbiota need to be changed in order to benefit the liver and/or CVD risk. A recent phase-2 controlled trial involving 104 patients with NAFLD examined whether administration of a synbiotic combination of probiotic and prebiotic agents for 1 year affected liver fat content, non-invasive fibrosis scores, liver stiffness and the composition of the faecal microbiome [108]. The results of this trial showed that the synbiotic did not significantly improve liver fat content, liver stiffness, or liver fibrosis scores. However, the synbiotic significantly altered the faecal microbiome, but it is uncertain whether these changes in microbiota had any beneficial effects on the vascular system and CVD risk [108].

Collectively, as discussed above, there are likely multiple underlying mechanisms by which NAFLD can directly increase the risk of CVD and cardiac/arrhythmic complications. Indeed, NAFLD exacerbates insulin resistance, promotes atherogenic dyslipidaemia, induces hypertension and causes the synthesis of several pro- 
atherogenic, pro-coagulant and pro-inflammatory mediators that may contribute to the development of CVD and other cardiac/arrhythmic complications. Nevertheless, other mechanistic studies are needed to better elucidate the existing but complex link between NAFLD and these cardiovascular complications. In particular, more research is needed to investigate the role of low-grade systemic inflammation in the development of myocardial remodeling (cardiomyopathy), as well as cardiac autonomic abnormalities and myocardial electrical instability (arrhythmias) associated with NAFLD.

\section{CVD RISK PREDICTION AND LIFESTYLE/PHARMACEUTICAL MODIFIERS OF NAFLD-RELATED CVD RISK}

\subsection{CVD risk assessment}

In adults with NAFLD, for the primary prevention of CVD, determining the individual's risk of future CVD events is essential to successfully implementing CVD risk reduction [71]. Several algorithms are commonly used in the general population to estimate one's future risk of CVD events and can be applied to adults with NAFLD. These include the Framingham Risk score (FRS) and the Atherosclerotic Cardiovascular Disease (ASCVD) score [109]. The FRS incorporates age, blood pressure, total cholesterol, HDL-cholesterol, smoking, and pre-existing T2DM to estimate an individual's 10-year risk of coronary heart disease (CHD) [110]. Treeprasertsuk et al. found that the FRS accurately predicted the 10-year CHD risk in adults with NAFLD [111]. Further, they noted that adults with NAFLD had a higher predicted 10-year risk of CHD as assessed by the FRS than age- and sex-matched control individuals. The ASCVD risk score is an alternative algorithm that predicts 10 -year risk of CHD by incorporating age, race, sex, blood pressure, plasma lipids, diabetes, smoking, and use of anti-hypertensive drugs, statins or aspirin [112]. The ASCVD risk score is also a predictor of major adverse CVD events in adults with NAFLD and can be used to estimate future CVD risk in this patient population (unpublished data, KEC). That said, further studies are needed to ascertain whether a risk multiplier should be applied to NAFLD patients over and above other CVD risk factors already included in these risk algorithms, or whether addition of NAFLD may improve the accuracy of risk algorithms to predict adverse CVD outcomes.

In select cases, coronary computed tomography angiogram might be beneficial in CVD risk assessment in adults with NAFLD. Recently, Meyersohn et al. evaluated the prognostic impact of fatty liver (detected by coronary computed tomography angiogram) on prevalent CHD and incident major CVD events in a nested cohort study of 3,756 United States middle-aged individuals followed for a median period of 25 months. At baseline, compared to those without fatty liver on cardiac imaging, adults with fatty liver had higher coronary artery calcium scores, higher segment involvement scores and greater Leaman's scores (i.e., a composite measure of extent and severity of atherosclerotic plaques) [113]. In addition, for every class of coronary artery disease (i.e., no significant CAD, non-obstructive CAD or obstructive CAD) on baseline coronary computed tomography angiogram, the addition of fatty liver was associated with a higher risk of CVD events than those with the same degree of CAD but without fatty liver [113]. Thus, in adults with NAFLD, coronary computed tomography scans might provide valuable information to aid in CVD risk prediction.

Finally, the presence of advanced liver fibrosis is associated with higher CVD risk in adults with NAFLD. As mentioned previously, Henson et al. reported that in adults with 
NAFLD, advanced fibrosis (fibrosis stage 3-4) on histology and by non-invasive testing (e.g., Fibrosis-4 score) was independently associated with increased risk of incident CVD events [21]. Thus, clinicians should have a high degree of suspicion for CVD in NAFLD adults with advanced fibrosis.

\subsection{Modifying NAFLD-related CVD risk: lifestyle intervention}

Once CVD risk is assessed, lifestyle interventions including hypocaloric diet and moderate physical exercise should be always implemented to both reduce CVD risk and for the treatment of NAFLD (Figure 4).

Physical activity is recommended for all individuals, regardless of NAFLD, to improve risk of CVD. Seventy-five minutes of vigorous physical activity or 150 minutes of moderate intensity activity weekly is associated with CVD risk reduction [109, 114]. Physical activity is also beneficial for treatment of NAFLD. A recent meta-analysis of exercise for NAFLD (predominantly aerobic exercise) found that even in the absence of weight loss or dietary changes, physical activity may decrease hepatic steatosis and may improve serum aminotransferase levels [115]. Thus, weekly exercise can both improve NAFLD and decreased CVD risk. It is essential to note that any degree of physical activity is considered beneficial and for those patients who are unable to exercise to the duration and intensity goals above, regular exercise of any intensity and duration should be encouraged.

The Mediterranean diet is also beneficial for both NAFLD and CVD risk reduction. The Mediterranean diet focuses on limiting consumption of red and processed meats while increasing consumption of vegetables, legumes or fruit. Whole grains should be minimally processed, and the primary sources of fat should be $n-3$ fatty acids, such as fish, olive oil and nuts. A large randomized trial found that adults at high risk of CVD assigned to two forms of the Mediterranean diet (Mediterranean diet supplemented with olive oil or mixed nuts) had a lower incidence of CVD events than those advised to follow a low fat diet [116]. Increasing evidence also supports the value of the Mediterranean diet for treatment of NAFLD. Relative to a low fat, high carbohydrate diet, the Mediterranean diet leads to a greater decrease in insulin resistance and fatty liver [117]. Results from the Framingham Heart Study also found that the Mediterranean diet was associated with a lower incidence of fatty liver and decreased severity of fatty liver [118].

Tobacco use is an important contributor to CVD risk and premature death [119]. Further, tobacco use may contribute to liver fibrosis progression and poor outcomes in NAFLD [21, 120]. Current guidelines recommend that clinicians routinely screen for tobacco use and refer patients for both behavorial and pharmacotherapy interventions to aid smoking cessation [109, 114].

Dyslipidaemia, hypertension, T2DM and the MetS are established CVD risk factors and frequently occur concurrently in NAFLD $[109,114]$. Thus, primary care physicians are strongly encouarged to screen for and treat these conditions to modify CVD risk (as summarized in Figure 4).

\subsection{Modifying NAFLD-related CVD risk: pharmacotherapy}


Presently, there are no approved pharmacological treatments for NAFLD. The cornerstone of NAFLD management remains lifestyle modification. In this section, we specifically discuss the current pharmacological treatments for modifying the NAFLDrelated CVD risk.

Daily aspirin use is strongly recommended for adults with a prior history of CVD as well as for adults aged 40-70 years at high CVD risk but not at increased bleeding risk [109, 114]. In adults with a low risk of CVD or high risk of bleeding, the CVD benefits drived from aspirin do not outweigh the risk of bleeding and thus aspirin in not recommended. In a cohort of adults with histologically-defined NAFLD, aspirin use was associated with a lower risk of advanced liver fibrosis that increased as the duration of aspirin use increased. Importantly, the use of non-aspirin non-steroidal anti-inflammatory drugs did not impact liver fibrosis [121]. Thus, for NAFLD patients with a CVD indication for aspirin, especially those with a history of CVD, daily aspirin therapy should be strongly considered.

Several clinical trials have shown that the chronic use of statins markedly reduces the risk CVD events and all-cause mortality [122]. Importantly, statins are also safe to use in adults with NAFLD, but are often under-utilized [123]. We strongly recommend initiation of statin therapy for any patients with CVD indications, regardless of NAFLD. However, there is insufficient evidence to recommend statins to specifically treat NAFLD. That said, there is limited evidence that chronic use of statins may also be associated with a reduced risk of hepatocellular carcinoma [124].

Glucagon-like peptide-1 receptor agonists (GLP-1 RAs) increase insulin production from pancreatic beta cells as well as muscle and adipose glucose uptakes while decreasing glucose production in the liver leading to decreased plasma glucose levels in adults with T2DM. Further, GLP-1 RAs decrease satiety and reduce gastric emptying leading to weight loss. Several GLP-1 RAs have been shown to exert beneficial CVD effects in adults with T2DM. For instance, in the Liraglutide Effect and Action in Diabetes: Evaluation of Cardiovascular Outcome Results (LEADER) trials, liraglutide significantly reduced CVD events, CVD-related mortality and all-cause mortality compared to placebo [125]. Semaglutide, as a weekly injection, also reduced CVD events, largely driven by a reduction in ischemic strokes compared to placebo, while dulaglutide reduced both CVD events and CVD-related deaths [126, 127]. At present, it remains debatable if the aforementioned CVD benefits are a class effect, because other GLP-1 RAs have shown borderline or no benefit in CVD event reduction [128, 129]. Data on the impact of GLP-1 agonists on arrhythmias is limited with a metaanalysis showing no association with GLP-1 agonists and permanent AF [130], and a study in rats showing reduced ventricular arrhythmic potential with administration of exendin-4 [131].

GLP-1 RAs may also be beneficial in NAFLD. In a phase 2 randomized controlled trial (the LEAN trial) that involved 52 obese patients with $\mathrm{NASH}$, liraglutide $1.8 \mathrm{mg}$ daily resulted in significantly higher rates of NASH resolution over 48 weeks when compared to placebo [132], although it remains uncertain whether benefit is mediated by weight loss with this agent. Currently, semaglutide is being evaluated for NASH treatment and an analysis of a phase 2 randomized controlled trial of semaglutide vs. placebo for $\mathrm{NASH}$ fibrosis stage 2-3 found that the highest dose of daily subcutaneous semaglutide $0.4 \mathrm{mg}$ resulted in more frequent $\mathrm{NASH}$ resolution than placebo (59\% vs. $17 \%$, 
unpublished data). Randomized controlled trials assessing the effect of semaglutide (NCT02970942, enrollment completed) or dulaglutide (NCT03648554) on NASH histology are currently ongoing.

Sodium-glucose cotransporter-2 (SGLT2) inhibitors act in the renal proximal tubule to prevent glucose reabsorption, reducing plasma glucose levels and causing glycosuria as well as diuresis, leading to reduction in blood pressure and body weight. In adults with established T2DM, empagliflozin was also associated with a significant reduction in the composite endpoint of myocardial infarction, stroke or CVD-related deaths, as well as reduction in hospital admissions for heart failure and all-cause mortality [133]. Canagliflozin in adults with T2DM also reduced a composite CVD event outcome and reduced heart failure admissions when compared to placebo and may have benefits in prevent progression of renal disease [134]. Further, SGLT2 may also be effecting in preventing or treating AF [135]. In a murine model of diabetes induced by high fat diet and low-dose streptomycin injection, Shao et al. reported that compared to the control group, diabetic rats had increased mitochondrial dysfunction and greater AF inducibility, suggesting an important role for mitochondrial dysfunction in the pathogenesis of AF in diabetes [136]. In this study, treatment with empagliflozin improved mitochondrial dysfunction and biogenesis, as well as atrial electrical and structural remodelling, supporting a possible beneficial role of empagliflozin for AF prevention in diabetes.

Both empagliflozin and canagliflozin have shown promise in the treatment of NAFLD. For instance, in adults with T2DM, 20 weeks of empagliflozin reduced hepatic fat content and reduced serum aminotransferase levels when compared to placebo [137]. In a single arm, pilot trial of 24 weeks empagliflozin in adults with well-controlled T2DM demonstrated a reduction in hepatic steatosis, hepatocyte ballooning and fibrosis compared to a historic control group [138]. Recently, other two randomized controlled trials assessed the impact of empagliflozin vs. placebo on liver fat assessed by MRI. The first study, among adults with NAFLD and well controlled T2DM, found that empagliflozin for 24 weeks significantly lowered liver fat content by MRI and body weight [139]. The second study, conducted in those without T2DM, found that, compared to placebo, 24 weeks of empagliflozin caused a significant decrease in liver fibrosis as assessed by transient elastography (Fibroscan ${ }^{\circledR}$ ), although there was no difference in hepatic fat as assessed by controlled attenuated parameter [140]. A recent trial evaluated the impact of canagliflozin vs. placebo on liver fat levels. Canagliflozin reduced liver fat levels, a reduction that strongly correlated with weight loss and also improved hepatic insulin sensitivity [141]. As GLP-1 RAs and SGLT2inhibitors have proven benefits in CVD and may also have benefits in NAFLD, not least to facilitate weight loss, we recommend consideration of these newer antihyperglycaemic drugs (alone or in combination with metformin) for most patients with T2DM and NAFLD.

It is known that use of pioglitazone in NASH patients has beneficial effects on serum liver enzyme levels, liver fat and resolution of NASH both in patients with and without T2DM [142-145]. When compared to its beneficial effects on NASH, the effect of pioglitazone on the histological severity of liver fibrosis is modest. However, in a trial of 101 patients with NASH and prediabetes or T2DM, who were randomly assigned to pioglitazone or placebo for 18 months, Cusi et al. reported that long-term pioglitazone 
treatment was safe and effective in patients with prediabetes or T2DM and NASH [146]. Pioglitazone treatment also improved fibrosis stage and all other individual histologic scores of NASH. Interestingly, in a meta-analysis of 8 randomized trials, Musso et al. showed that pioglitazone treatment reduced advanced liver fibrosis in patients with $\mathrm{NASH}$, irrespective of T2DM status [147]. Although pioglitazone is not currently approved outside the treatment for T2DM, the American and European guidelines for management of NAFLD recommended the use of pioglitazone in adults with biopsyconfirmed NASH, regardless of diabetes status [148, 149]. However, concerns about weight gain, fluid retention and risk of bone fractures may restrict the wider clinical use of pioglitazone in NAFLD patients. That said, taking into consideration that it is known that pioglitazone may also exert some cardiovascular benefits in patients with T2DM or prediabetes, this inexpensive, generic medication should also be considered in $\mathrm{NASH}$ patients without a history of congestive heart failure [150-152].

\subsection{Impact of histologic improvement in NAFLD on CVD risk}

While NAFLD is a risk factor for CVD, the impact of histologic improvement or resolution of NASH on CVD risk remains currently unknown [10]. A post-hoc analysis of the PIVENS trial found that patients with NASH resolution, independent of treatment arm, had significant improvements in serum trigylceride and HDL-C levels, as well as a decrease in the FRS when compared to those without NASH resolution [153]. A subsequent analysis of this trial also demonstrated that patients with NASH resolution showed a favorable increase in LDL particle size and an increase in the prevalence of LDL phenotype A, both associated with less atherogenic LDL particles and decreased CVD risk [154]. Thus, while the impact of NASH resolution on CVD risk is not yet known, resolution of NASH is associated with favorable changes both in lipid risk factors for CVD and in the FRS.

\subsection{CVD testing and referral}

For all adults with NAFLD and new-onset symptoms concerning for CVD, additional testing, including carotid ultrasonography, electrocardiogram, transthoracic echocardiography and cardiac stress testing can be performed and referral to a cardiologist is highly recommended. Coronary computed tomography angiography can also be considered for those unable to participate in exercise stress testing or with indeterminate stress testing results [155].

Collectively, whereas we acknowledge that any future decision on approval of new therapies for NAFLD is based upon the histological improvements in NASH and/or stage of liver fibrosis, we want to draw particular attention to the possible cardiometabolic effects of these newer drugs. Indeed, it is clinically important to understand how new therapies for NAFLD may impact the CVD risk profile in these patients [156]. The ideal therapeutic drug(s) in NAFLD would not only ameliorate liver disease but also prevent cardiovascular and cardiac/arrhythmic complications associated with NAFLD.

\section{CONCLUSIONS}

The current evidence strongly supports the notion that NAFLD is a 'multisystem' disease that adversely affects many extra-hepatic organ systems, including the cardiovascular system [10]. NAFLD independently increases the risk of coronary 
atherosclerosis, cardiomyopathy and arrhythmias, which clinically result in increased CVD morbidity and mortality. This risk parallels the severity of NAFLD (especially the stage of liver fibrosis). Although further studies are needed to definitely establish the existence of an independent contribution of NAFLD to risk of developing adverse CVD outcomes and other cardiac/arrhythmic complications, all these observations may have important implications for decision-making in clinical practice and public health [10]. Indeed, all patients with NAFLD on primary prevention of CVD should undergo careful cardiovascular surveillance (at least by the use of algorithms for CVD risk prediction) and aggressive pharmacological treatment of coexisting CVD risk factors should be considered in NAFLD patients, as these patients are at higher risk for CVD events.

CONFLICT OF INTEREST STATEMENT: All the authors do not have any conflict of interest to disclose.

FUNDING SOURCES: GT is supported in part by grants from the School of Medicine, University of Verona, Verona, Italy. CDB is supported in part by the Southampton NIHR Biomedical Research Centre (IS-BRC-20004), UK.

\section{REFERENCES}

1. Paik JM, Golabi P, Younossi Y, Mishra A, Younossi ZM. Changes in the Global Burden of Chronic Liver Diseases From 2012 to 2017: The Growing Impact of Nonalcoholic Fatty Liver Disease. Hepatology. 2020. Epub 2020/02/12. doi: 10.1002/hep.31173. PubMed PMID: 32043613.

2. Mantovani A, Scorletti E, Mosca A, Alisi A, Byrne CD, Targher G. Complications, morbidity and mortality of nonalcoholic fatty liver disease. Metabolism. 2020:154170. Epub 2020/02/02. doi: 10.1016/j.metabol.2020.154170. PubMed PMID: 32006558.

3. Targher G, Day CP, Bonora E. Risk of cardiovascular disease in patients with nonalcoholic fatty liver disease. N Engl J Med. 2010;363(14):1341-50. Epub 2010/10/01. doi: 10.1056/NEJMra0912063. PubMed PMID: 20879883.

4. Adams LA, Anstee QM, Tilg H, Targher G. Non-alcoholic fatty liver disease and its relationship with cardiovascular disease and other extrahepatic diseases. Gut. 2017;66(6):1138-53. Epub 2017/03/21. doi: 10.1136/gutjnl-2017-313884. PubMed PMID: 28314735.

5. Younossi ZM, Blissett D, Blissett R, Henry L, Stepanova M, Younossi Y, et al. The economic and clinical burden of nonalcoholic fatty liver disease in the United States and Europe. Hepatology. 2016;64(5):1577-86. Epub 2016/10/22. doi: 10.1002/hep.28785. PubMed PMID: 27543837.

6. Byrne CD, Targher G. NAFLD: a multisystem disease. J Hepatol. 2015;62(1 Suppl):S47-64. Epub 2015/04/29. doi: 10.1016/j.jhep.2014.12.012. PubMed PMID: 25920090.

7. Byrne CD, Targher G. NAFLD as a driver of chronic kidney disease. J Hepatol. 2020;72(4):785-801. Epub 2020/02/16. doi: 10.1016/j.jhep.2020.01.013. PubMed PMID: 32059982.

8. Anstee QM, Mantovani A, Tilg H, Targher G. Risk of cardiomyopathy and cardiac arrhythmias in patients with nonalcoholic fatty liver disease. Nat Rev Gastroenterol Hepatol. 2018;15(7):425-39. Epub 2018/05/02. doi: 10.1038/s41575-018-0010-0. PubMed PMID: 29713021.

9. Paik JM, Henry L, De Avila L, Younossi E, Racila A, Younossi ZM. Mortality Related to Nonalcoholic Fatty Liver Disease Is Increasing in the United States. Hepatol Commun. 2019;3(11):1459-71. Epub 2019/11/09. doi: 10.1002/hep4.1419. PubMed PMID: 31701070; PubMed Central PMCID:

PMCPMC6824058. 
10. Targher G, Byrne CD, Tilg H. NAFLD and increased risk of cardiovascular disease: clinical associations, pathophysiological mechanisms and pharmacological implications. Gut. 2020;69(9):1691-705. Epub 2020/04/24. doi: 10.1136/gutjnl-2020-320622. PubMed PMID: 32321858.

11. Zhou YY, Zhou XD, Wu SJ, Fan DH, Van Poucke S, Chen YP, et al. Nonalcoholic fatty liver disease contributes to subclinical atherosclerosis: A systematic review and meta-analysis. Hepatol Commun. 2018;2(4):376-92. Epub 2018/04/06. doi: 10.1002/hep4.1155. PubMed PMID: 29619417; PubMed Central PMCID: PMCPMC5880194.

12. Targher G, Byrne CD, Lonardo A, Zoppini G, Barbui C. Non-alcoholic fatty liver disease and risk of incident cardiovascular disease: A meta-analysis. J Hepatol. 2016;65(3):589-600. Epub 2016/05/24. doi: 10.1016/j.jhep.2016.05.013. PubMed PMID: 27212244.

13. Sinn DH, Kang D, Chang Y, Ryu S, Cho SJ, Paik SW, et al. Non-alcoholic fatty liver disease and the incidence of myocardial infarction: A cohort study. J Gastroenterol Hepatol. 2020;35(5):833-9. Epub 2019/09/13. doi: 10.1111/jgh.14856. PubMed PMID: 31512278.

14. Labenz C, Huber Y, Michel M, Nagel M, Galle PR, Kostev K, et al. Impact of NAFLD on the Incidence of Cardiovascular Diseases in a Primary Care Population in Germany. Dig Dis Sci. 2020;65(7):2112-9. Epub 2019/12/05. doi: 10.1007/s10620-019-05986-9. PubMed PMID: 31797186.

15. Alexander M, Loomis AK, van der Lei J, Duarte-Salles T, Prieto-Alhambra D, Ansell D, et al. Nonalcoholic fatty liver disease and risk of incident acute myocardial infarction and stroke: findings from matched cohort study of 18 million European adults. BMJ. 2019;367:15367. Epub 2019/10/09. doi: 10.1136/bmj.15367. PubMed PMID: 31594780; PubMed Central PMCID: PMCPMC6780322..

16. Sinn DH, Kang D, Chang Y, Ryu S, Gu S, Kim H, et al. Non-alcoholic fatty liver disease and progression of coronary artery calcium score: a retrospective cohort study. Gut. 2017;66(2):323-9. Epub 2016/09/08. doi: 10.1136/gutjnl-2016-311854. PubMed PMID: 27599521.

17. Sinn DH, Cho SJ, Gu S, Seong D, Kang D, Kim H, et al. Persistent Nonalcoholic Fatty Liver Disease Increases Risk for Carotid Atherosclerosis. Gastroenterology. 2016;151(3):481-8 e1. Epub 2016/06/11. doi: 10.1053/j.gastro.2016.06.001. PubMed PMID: 27283259.

18. Hagstrom H, Nasr P, Ekstedt M, Hammar U, Stal P, Hultcrantz R, et al. Fibrosis stage but not NASH predicts mortality and time to development of severe liver disease in biopsy-proven NAFLD. J Hepatol. 2017;67(6):1265-73. Epub 2017/08/15. doi: 10.1016/j.jhep.2017.07.027. PubMed PMID: 28803953.

19. Taylor RS, Taylor RJ, Bayliss S, Hagstrom H, Nasr P, Schattenberg JM, et al. Association Between Fibrosis Stage and Outcomes of Patients With Nonalcoholic Fatty Liver Disease: A Systematic Review and Meta-Analysis. Gastroenterology. 2020;158(6):1611-25 e12. Epub 2020/02/07. doi:

10.1053/j.gastro.2020.01.043. PubMed PMID: 32027911.

20. Vilar-Gomez E, Calzadilla-Bertot L, Wai-Sun Wong V, Castellanos M, Aller-de la Fuente R, Metwally $M$, et al. Fibrosis Severity as a Determinant of Cause-Specific Mortality in Patients With Advanced Nonalcoholic Fatty Liver Disease: A Multi-National Cohort Study. Gastroenterology. 2018;155(2):443-57 e17. Epub 2018/05/08. doi: 10.1053/j.gastro.2018.04.034. PubMed PMID: 29733831.

21. Henson JB, Simon TG, Kaplan A, Osganian S, Masia R, Corey KE. Advanced fibrosis is associated with incident cardiovascular disease in patients with non-alcoholic fatty liver disease. Aliment Pharmacol Ther. 2020. Epub 2020/02/12. doi: 10.1111/apt.15660. PubMed PMID: 32043602.

22. Mantovani A, Dauriz M, Sandri D, Bonapace S, Zoppini G, Tilg H, et al. Association between nonalcoholic fatty liver disease and risk of atrial fibrillation in adult individuals: An updated meta-analysis. Liver Int. 2019;39(4):758-69. Epub 2019/01/19. doi: 10.1111/liv.14044. PubMed PMID: 30657626.

23. Cai X, Zheng S, Liu Y, Zhang Y, Lu J, Huang Y. Nonalcoholic fatty liver disease is associated with increased risk of atrial fibrillation. Liver Int. 2020;40(7):1594-600. Epub 2020/04/13. doi: 10.1111/liv.14461. PubMed PMID: 32279432.

24. Park HE, Lee H, Choi SY, Kim HS, Chung GE. The risk of atrial fibrillation in patients with nonalcoholic fatty liver disease and a high hepatic fibrosis index. Sci Rep. 2020;10(1):5023. Epub 2020/03/21. doi: 10.1038/s41598-020-61750-4. PubMed PMID: 32193478; PubMed Central PMCID: PMCPMC7081198. 25. Hung CS, Tseng PH, Tu CH, Chen CC, Liao WC, Lee YC, et al. Nonalcoholic Fatty Liver Disease Is Associated With QT Prolongation in the General Population. J Am Heart Assoc. 2015;4(7). Epub 2015/07/23. doi: 10.1161/JAHA.115.001820. PubMed PMID: 26199227; PubMed Central PMCID: PMCPMC4608068.

26. Mantovani A, Rigamonti A, Bonapace S, Bolzan B, Pernigo M, Morani G, et al. Nonalcoholic Fatty Liver Disease Is Associated With Ventricular Arrhythmias in Patients With Type 2 Diabetes Referred for Clinically Indicated 24-Hour Holter Monitoring. Diabetes Care. 2016;39(8):1416-23. Epub 2016/05/26. doi: 10.2337/dc16-0091. PubMed PMID: 27222503. 
27. Mangi MA, Minhas AM, Rehman H, Pathan F, Liang H, Beidas S. Association of Non-alcoholic Fatty Liver Disease with Conduction Defects on Electrocardiogram. Cureus. 2017;9(3):e1107. Epub 2017/04/26. doi: 10.7759/cureus.1107. PubMed PMID: 28439482; PubMed Central PMCID: PMCPMC5400518.

28. Mantovani A, Rigolon R, Pichiri I, Bonapace S, Morani G, Zoppini G, et al. Nonalcoholic fatty liver disease is associated with an increased risk of heart block in hospitalized patients with type 2 diabetes mellitus. PLoS One. 2017;12(10):e0185459. Epub 2017/10/06. doi: 10.1371/journal.pone.0185459. PubMed PMID: 28981521; PubMed Central PMCID: PMCPMC5628831.

29. Wijarnpreecha K, Lou S, Panjawatanan P, Cheungpasitporn W, Pungpapong S, Lukens FJ, et al. Association between diastolic cardiac dysfunction and nonalcoholic fatty liver disease: A systematic review and meta-analysis. Dig Liver Dis. 2018;50(11):1166-75. Epub 2018/10/08. doi: 10.1016/j.dld.2018.09.004. PubMed PMID: 30292566.

30. Chiu LS, Pedley A, Massaro JM, Benjamin EJ, Mitchell GF, McManus DD, et al. The association of non-alcoholic fatty liver disease and cardiac structure and function-Framingham Heart Study. Liver Int. 2020. Epub 2020/07/13. doi: 10.1111/liv.14600. PubMed PMID: 32654390.

31. Chung GE, Lee JH, Lee H, Kim MK, Yim JY, Choi SY, et al. Nonalcoholic fatty liver disease and advanced fibrosis are associated with left ventricular diastolic dysfunction. Atherosclerosis. 2018;272:13744. Epub 2018/04/01. doi: 10.1016/j.atherosclerosis.2018.03.027. PubMed PMID: 29604480.

32. Lee YH, Kim KJ, Yoo ME, Kim G, Yoon HJ, Jo K, et al. Association of non-alcoholic steatohepatitis with subclinical myocardial dysfunction in non-cirrhotic patients. J Hepatol. 2018;68(4):764-72. Epub 2017/11/28. doi: 10.1016/j.jhep.2017.11.023. PubMed PMID: 29175242.

33. Petta S, Argano C, Colomba D, Camma C, Di Marco V, Cabibi D, et al. Epicardial fat, cardiac geometry and cardiac function in patients with non-alcoholic fatty liver disease: association with the severity of liver disease. J Hepatol. 2015;62(4):928-33. Epub 2014/12/03. doi: 10.1016/j.jhep.2014.11.030. PubMed PMID: 25445395.

34. Sunbul M, Kivrak T, Durmus E, Akin H, Aydin Y, Ergelen R, et al. Nonalcoholic Steatohepatitis Score is an Independent Predictor of Right Ventricular Dysfunction in Patients with Nonalcoholic Fatty Liver Disease. Cardiovasc Ther. 2015;33(5):294-9. Epub 2015/07/24. doi: 10.1111/1755-5922.12145. PubMed PMID: 26202098.

35. Simon TG, Bamira DG, Chung RT, Weiner RB, Corey KE. Nonalcoholic Steatohepatitis is Associated with Cardiac Remodeling and Dysfunction. Obesity (Silver Spring). 2017;25(8):1313-6. Epub 2017/07/27. doi: 10.1002/oby.21879. PubMed PMID: 28745025; PubMed Central PMCID: PMCPMC5648006.

36. Volzke H, Haring R, Lorbeer R, Wallaschofski $\mathrm{H}$, Reffelmann $\mathrm{T}$, Empen $\mathrm{K}$, et al. Heart valve sclerosis predicts all-cause and cardiovascular mortality. Atherosclerosis. 2010;209(2):606-10. Epub 2009/11/20. doi: 10.1016/j.atherosclerosis.2009.10.030. PubMed PMID: 19922935.

37. Rossi A, Targher G, Zoppini G, Cicoira M, Bonapace S, Negri C, et al. Aortic and mitral annular calcifications are predictive of all-cause and cardiovascular mortality in patients with type 2 diabetes. Diabetes Care. 2012;35(8):1781-6. Epub 2012/06/16. doi: 10.2337/dc12-0134. PubMed PMID: 22699285; PubMed Central PMCID: PMCPMC3402245.

38. Di Minno MN, Di Minno A, Ambrosino P, Songia P, Tremoli E, Poggio P. Aortic valve sclerosis as a marker of atherosclerosis: Novel insights from hepatic steatosis. Int J Cardiol. 2016;217:1-6. Epub 2016/05/11. doi: 10.1016/j.ijcard.2016.04.162. PubMed PMID: 27164417.

39. Mantovani A, Pernigo M, Bergamini C, Bonapace S, Lipari P, Valbusa F, et al. Heart valve calcification in patients with type 2 diabetes and nonalcoholic fatty liver disease. Metabolism. 2015;64(8):879-87. Epub 2015/05/11. doi: 10.1016/j.metabol.2015.04.003. PubMed PMID: 25957758.

40. Targher G, Lonardo A, Byrne CD. Nonalcoholic fatty liver disease and chronic vascular complications of diabetes mellitus. Nat Rev Endocrinol. 2018;14(2):99-114. Epub 2017/12/30. doi:

10.1038/nrendo.2017.173. PubMed PMID: 29286050.

41. Mantovani A, Byrne CD, Bonora E, Targher G. Nonalcoholic Fatty Liver Disease and Risk of Incident Type 2 Diabetes: A Meta-analysis. Diabetes Care. 2018;41(2):372-82. doi: 10.2337/dc17-1902. PubMed PMID: 29358469.

42. Lonardo A, Nascimbeni F, Mantovani A, Targher G. Hypertension, diabetes, atherosclerosis and NASH: Cause or consequence? J Hepatol. 2018;68(2):335-52. Epub 2017/11/11. doi: 10.1016/j.jhep.2017.09.021. PubMed PMID: 29122390.

43. Wild SH, Walker JJ, Morling JR, McAllister DA, Colhoun HM, Farran B, et al. Cardiovascular Disease, Cancer, and Mortality Among People With Type 2 Diabetes and Alcoholic or Nonalcoholic Fatty Liver 
Disease Hospital Admission. Diabetes Care. 2018;41(2):341-7. Epub 2017/11/24. doi: 10.2337/dc17-1590. PubMed PMID: 29167212.

44. Wainwright $P$, Scorletti E, Byrne CD. Type 2 Diabetes and Hepatocellular Carcinoma: Risk Factors and Pathogenesis. Current diabetes reports. 2017;17(4):20. Epub 2017/03/16. doi: 10.1007/s11892-0170851-x. PubMed PMID: 28290049.

45. Alexander M, Loomis AK, van der Lei J, Duarte-Salles T, Prieto-Alhambra D, Ansell D, et al. Risks and clinical predictors of cirrhosis and hepatocellular carcinoma diagnoses in adults with diagnosed NAFLD: real-world study of 18 million patients in four European cohorts. BMC Med. 2019;17(1):95. Epub 2019/05/21. doi: 10.1186/s12916-019-1321-x. PubMed PMID: 31104631; PubMed Central PMCID: PMCPMC6526616.

46. Patel YA, Gifford EJ, Glass LM, McNeil R, Turner MJ, Han B, et al. Risk factors for biopsy-proven advanced non-alcoholic fatty liver disease in the Veterans Health Administration. Aliment Pharmacol Ther. 2018;47(2):268-78. Epub 2017/11/09. doi: 10.1111/apt.14411. PubMed PMID: 29115682; PubMed Central PMCID: PMCPMC5861349.

47. Angulo $P$, Keach JC, Batts KP, Lindor KD. Independent predictors of liver fibrosis in patients with nonalcoholic steatohepatitis. Hepatology. 1999;30(6):1356-62. Epub 1999/11/26. doi:

10.1002/hep.510300604. PubMed PMID: 10573511.

48. Sung KC, Jeong WS, Wild SH, Byrne CD. Combined influence of insulin resistance, overweight/obesity, and fatty liver as risk factors for type 2 diabetes. Diabetes Care. 2012;35(4):717-22. doi: dc11-1853 [pii];10.2337/dc11-1853 [doi].

49. Zhou YY, Zhou XD, Wu SJ, Hu XQ, Tang B, Poucke SV, et al. Synergistic increase in cardiovascular risk in diabetes mellitus with nonalcoholic fatty liver disease: a meta-analysis. European journal of gastroenterology \& hepatology. 2018;30(6):631-6. Epub 2018/01/20. doi:

10.1097/meg.0000000000001075. PubMed PMID: 29351115.

50. Katsiki N, Mikhailidis DP, Mantzoros CS. Non-alcoholic fatty liver disease and dyslipidemia: An update. Metabolism. 2016;65(8):1109-23. Epub 2016/05/31. doi: 10.1016/j.metabol.2016.05.003. PubMed PMID: 27237577.

51. Chatrath $H$, Vuppalanchi $R$, Chalasani N. Dyslipidemia in patients with nonalcoholic fatty liver disease. Seminars in liver disease. 2012;32(1):22-9. doi: 10.1055/s-0032-1306423.

52. Castillo-Leon E, Connelly MA, Konomi JV, Caltharp S, Cleeton R, Vos MB. Increased atherogenic lipoprotein profile in children with non-alcoholic steatohepatitis. Pediatric obesity. 2020:e12648. Epub 2020/05/06. doi: 10.1111/ijpo.12648. PubMed PMID: 32367624.

53. Lim S, Park YM, Sakuma I, Koh KK. How to control residual cardiovascular risk despite statin treatment: focusing on HDL-cholesterol. Int J Cardiol. 2013;166(1):8-14. Epub 2012/04/17. doi: 10.1016/j.ijcard.2012.03.127. PubMed PMID: 22503572.

54. Graham I, Cooney MT, Bradley D, Dudina A, Reiner Z. Dyslipidemias in the prevention of cardiovascular disease: risks and causality. Current cardiology reports. 2012;14(6):709-20. Epub 2012/09/12. doi: 10.1007/s11886-012-0313-7. PubMed PMID: 22965836.

55. Tenenbaum A, Klempfner R, Fisman EZ. Hypertriglyceridemia: a too long unfairly neglected major cardiovascular risk factor. Cardiovascular diabetology. 2014;13:159. Epub 2014/12/05. doi: 10.1186/s12933-014-0159-y. PubMed PMID: 25471221; PubMed Central PMCID: PMCPMC4264548. 56. Nikolic D, Katsiki N, Montalto G, Isenovic ER, Mikhailidis DP, Rizzo M. Lipoprotein subfractions in metabolic syndrome and obesity: clinical significance and therapeutic approaches. Nutrients. 2013;5(3):928-48. Epub 2013/03/20. doi: 10.3390/nu5030928. PubMed PMID: 23507795; PubMed Central PMCID: PMCPMC3705327.

57. Sugino I, Kuboki K, Matsumoto T, Murakami E, Nishimura C, Yoshino G. Influence of fatty liver on plasma small, dense LDL- cholesterol in subjects with and without metabolic syndrome. Journal of atherosclerosis and thrombosis. 2011;18(1):1-7. Epub 2010/11/03. doi: 10.5551/jat.5447. PubMed PMID: 21041984.

58. Kikkawa K, Nakajima K, Shimomura Y, Tokita Y, Machida T, Sumino H, et al. Small dense LDL cholesterol measured by homogeneous assay in Japanese healthy controls, metabolic syndrome and diabetes patients with or without a fatty liver. Clinica chimica acta; international journal of clinical chemistry. 2015;438:70-9. Epub 2014/07/23. doi: 10.1016/j.cca.2014.07.017. PubMed PMID: 25050800.

59. Bril F, Sninsky JJ, Baca AM, Superko HR, Portillo Sanchez P, Biernacki D, et al. Hepatic Steatosis and Insulin Resistance, But Not Steatohepatitis, Promote Atherogenic Dyslipidemia in NAFLD. J Clin Endocrinol Metab. 2016;101(2):644-52. Epub 2015/12/18. doi: 10.1210/jc.2015-3111. PubMed PMID: 26672634. 
60. Imajo K, Hyogo H, Yoneda M, Honda Y, Kessoku T, Tomeno W, et al. LDL-migration index (LDL-MI), an indicator of small dense low-density lipoprotein (sdLDL), is higher in non-alcoholic steatohepatitis than in non-alcoholic fatty liver: a multicenter cross-sectional study. PLoS One. 2014;9(12):e115403. Epub 2014/12/30. doi: 10.1371/journal.pone.0115403. PubMed PMID: 25541989; PubMed Central PMCID: PMCPMC4277307.

61. Zhou Y, Llauradó G, Orešič M, Hyötyläinen T, Orho-Melander M, Yki-Järvinen H. Circulating triacylglycerol signatures and insulin sensitivity in NAFLD associated with the E167K variant in TM6SF2. J Hepatol. 2015;62(3):657-63. Epub 2014/12/03. doi: 10.1016/j.jhep.2014.10.010. PubMed PMID: 25457209. 62. Dongiovanni P, Petta S, Maglio C, Fracanzani AL, Pipitone R, Mozzi E, et al. Transmembrane 6 superfamily member 2 gene variant disentangles nonalcoholic steatohepatitis from cardiovascular disease. Hepatology. 2015;61(2):506-14. Epub 2014/09/25. doi: 10.1002/hep.27490. PubMed PMID: 25251399.

63. Kozlitina J, Smagris E, Stender S, Nordestgaard BG, Zhou HH, Tybjærg-Hansen A, et al. Exome-wide association study identifies a TM6SF2 variant that confers susceptibility to nonalcoholic fatty liver disease. Nature genetics. 2014;46(4):352-6. Epub 2014/02/18. doi: 10.1038/ng.2901. PubMed PMID: 24531328; PubMed Central PMCID: PMCPMC3969786.

64. Liu DJ, Peloso GM, Yu H, Butterworth AS, Wang X, Mahajan A, et al. Exome-wide association study of plasma lipids in >300,000 individuals. Nature genetics. 2017;49(12):1758-66. Epub 2017/10/31. doi: 10.1038/ng.3977. PubMed PMID: 29083408; PubMed Central PMCID: PMCPMC5709146.

65. Luukkonen PK, Nick A, Hölttä-Vuori M, Thiele C, Isokuortti E, Lallukka-Brück S, et al. Human PNPLA3-I148M variant increases hepatic retention of polyunsaturated fatty acids. JCI insight. 2019;4(16). Epub 2019/08/23. doi: 10.1172/jci.insight.127902. PubMed PMID: 31434800; PubMed Central PMCID: PMCPMC6777808.

66. Prill S, Caddeo A, Baselli G, Jamialahmadi O, Dongiovanni P, Rametta R, et al. The TM6SF2 E167K genetic variant induces lipid biosynthesis and reduces apolipoprotein $B$ secretion in human hepatic 3D spheroids. Scientific reports. 2019;9(1):11585. Epub 2019/08/14. doi: 10.1038/s41598-019-47737-w. PubMed PMID: 31406127; PubMed Central PMCID: PMCPMC6690969.

67. Brouwers M, Jacobs C, Bast A, Stehouwer CDA, Schaper NC. Modulation of Glucokinase Regulatory Protein: A Double-Edged Sword? Trends in molecular medicine. 2015;21(10):583-94. Epub 2015/10/04. doi: 10.1016/j.molmed.2015.08.004. PubMed PMID: 26432016.

68. Donnelly KL, Smith Cl, Schwarzenberg SJ, Jessurun J, Boldt MD, Parks EJ. Sources of fatty acids stored in liver and secreted via lipoproteins in patients with nonalcoholic fatty liver disease. J Clin Invest. 2005;115(5):1343-51. Epub 2005/05/03. doi: 10.1172/jci23621. PubMed PMID: 15864352; PubMed Central PMCID: PMCPMC1087172.

69. Simons P, Simons N, Stehouwer CDA, Schalkwijk CG, Schaper NC, Brouwers M. Association of common gene variants in glucokinase regulatory protein with cardiorenal disease: A systematic review and meta-analysis. PLoS One. 2018;13(10):e0206174. Epub 2018/10/24. doi: 10.1371/journal.pone.0206174. PubMed PMID: 30352097; PubMed Central PMCID: PMCPMC6198948.

70. Brouwers M, Simons N, Stehouwer CDA, Isaacs A. Non-alcoholic fatty liver disease and cardiovascular disease: assessing the evidence for causality. Diabetologia. 2020;63(2):253-60. Epub 2019/11/13. doi: 10.1007/s00125-019-05024-3. PubMed PMID: 31713012; PubMed Central PMCID: PMCPMC6946734.

71. Fricker ZP, Pedley A, Massaro JM, Vasan RS, Hoffmann U, Benjamin EJ, et al. Liver Fat Is Associated With Markers of Inflammation and Oxidative Stress in Analysis of Data From the Framingham Heart Study. Clinical gastroenterology and hepatology : the official clinical practice journal of the American Gastroenterological Association. 2019;17(6):1157-64.e4. Epub 2018/11/27. doi: 10.1016/j.cgh.2018.11.037. PubMed PMID: 30476583; PubMed Central PMCID: PMCPMC6475462.

72. Jiang F, Chen $Q$, Wang W, Ling Y, Yan Y, Xia P. Hepatocyte-derived extracellular vesicles promote endothelial inflammation and atherogenesis via microRNA-1. J Hepatol. 2020;72(1):156-66. Epub 2019/10/01. doi: 10.1016/j.jhep.2019.09.014. PubMed PMID: 31568800.

73. Meex RCR, Watt MJ. Hepatokines: linking nonalcoholic fatty liver disease and insulin resistance. Nat Rev Endocrinol. 2017;13(9):509-20. Epub 2017/06/09. doi: 10.1038/nrendo.2017.56. PubMed PMID: 28621339.

74. Lebensztejn DM, Flisiak-Jackiewicz M, Białokoz-Kalinowska I, Bobrus-Chociej A, Kowalska I. Hepatokines and non-alcoholic fatty liver disease. Acta Biochim Pol. 2016;63(3):459-67. Epub 2016/06/06. doi: 10.18388/abp.2016_1252. PubMed PMID: 27262842. 
75. Oh KJ, Lee DS, Kim WK, Han BS, Lee SC, Bae KH. Metabolic Adaptation in Obesity and Type II Diabetes: Myokines, Adipokines and Hepatokines. Int J Mol Sci. 2016;18(1). Epub 2016/12/22. doi: 10.3390/ijms18010008. PubMed PMID: 28025491; PubMed Central PMCID: PMCPMC5297643.

76. Jung TW, Yoo HJ, Choi KM. Implication of hepatokines in metabolic disorders and cardiovascular diseases. BBA Clin. 2016;5:108-13. Epub 2016/03/05. doi: 10.1016/j.bbacli.2016.03.002. PubMed PMID: 27051596; PubMed Central PMCID: PMCPMC4816030.

77. Stefan N, Häring HU. The role of hepatokines in metabolism. Nat Rev Endocrinol. 2013;9(3):144-52. Epub 2013/01/22. doi: 10.1038/nrendo.2012.258. PubMed PMID: 23337953.

78. Iroz A, Couty JP, Postic C. Hepatokines: unlocking the multi-organ network in metabolic diseases. Diabetologia. 2015;58(8):1699-703. Epub 2015/06/02. doi: 10.1007/s00125-015-3634-4. PubMed PMID: 26032022.

79. Fisher E, Stefan N, Saar K, Drogan D, Schulze MB, Fritsche A, et al. Association of AHSG gene polymorphisms with fetuin-A plasma levels and cardiovascular diseases in the EPIC-Potsdam study. Circ Cardiovasc Genet. 2009;2(6):607-13. Epub 2009/09/05. doi: 10.1161/CIRCGENETICS.109.870410. PubMed PMID: 20031641.

80. Yang SJ, Hwang SY, Choi HY, Yoo HJ, Seo JA, Kim SG, et al. Serum selenoprotein P levels in patients with type 2 diabetes and prediabetes: implications for insulin resistance, inflammation, and atherosclerosis. J Clin Endocrinol Metab. 2011;96(8):E1325-9. Epub 2011/06/15. doi: 10.1210/jc.2011-0620. PubMed PMID: 21677040.

81. Chow WS, Xu A, Woo YC, Tso AW, Cheung SC, Fong CH, et al. Serum fibroblast growth factor-21 levels are associated with carotid atherosclerosis independent of established cardiovascular risk factors. Arterioscler Thromb Vasc Biol. 2013;33(10):2454-9. Epub 2013/07/25. doi: 10.1161/ATVBAHA.113.301599. PubMed PMID: 23887638.

82. Hwang HJ, Jung TW, Hong HC, Seo JA, Kim SG, Kim NH, et al. LECT2 induces atherosclerotic inflammatory reaction via CD209 receptor-mediated JNK phosphorylation in human endothelial cells. Metabolism. 2015;64(9):1175-82. Epub 2015/06/06. doi: 10.1016/j.metabol.2015.06.001. PubMed PMID: 26123523.

83. Dongiovanni P, Meroni M, Baselli G, Mancina RM, Ruscica M, Longo $M$, et al. PCSK7 gene variation bridges atherogenic dyslipidemia with hepatic inflammation in NAFLD patients. J Lipid Res. 2019;60(6):1144-53. Epub 2019/03/29. doi: 10.1194/jlr.P090449. PubMed PMID: 30918065; PubMed Central PMCID: PMCPMC6547640.

84. Ipsen DH, Lykkesfeldt J, Tveden-Nyborg P. Molecular mechanisms of hepatic lipid accumulation in non-alcoholic fatty liver disease. Cellular and molecular life sciences : CMLS. 2018;75(18):3313-27. Epub 2018/06/25. doi: 10.1007/s00018-018-2860-6. PubMed PMID: 29936596; PubMed Central PMCID: PMCPMC6105174.

85. Parola M, Robino G. Oxidative stress-related molecules and liver fibrosis. J Hepatol. 2001;35(2):297306. Epub 2001/10/03. doi: 10.1016/s0168-8278(01)00142-8. PubMed PMID: 11580156.

86. Nati M, Haddad D, Birkenfeld AL, Koch CA, Chavakis T, Chatzigeorgiou A. The role of immune cells in metabolism-related liver inflammation and development of non-alcoholic steatohepatitis (NASH). Reviews in endocrine \& metabolic disorders. 2016;17(1):29-39. Epub 2016/02/06. doi: 10.1007/s11154-016-9339-2. PubMed PMID: 26847547.

87. Seki E, Schwabe RF. Hepatic inflammation and fibrosis: functional links and key pathways. Hepatology. 2015;61(3):1066-79. Epub 2014/07/30. doi: 10.1002/hep.27332. PubMed PMID: 25066777; PubMed Central PMCID: PMCPMC4306641.

88. Marí M, Caballero F, Colell A, Morales A, Caballeria J, Fernandez A, et al. Mitochondrial free cholesterol loading sensitizes to TNF- and Fas-mediated steatohepatitis. Cell Metab. 2006;4(3):185-98. Epub 2006/09/05. doi: 10.1016/j.cmet.2006.07.006. PubMed PMID: 16950136.

89. Pitisuttithum P, Chan WK, Goh GB, Fan JG, Song MJ, Charatcharoenwitthaya P, et al. Gammaglutamyl transferase and cardiovascular risk in nonalcoholic fatty liver disease: The Gut and Obesity Asia initiative. World J Gastroenterol. 2020;26(19):2416-26. Epub 2020/06/02. doi: 10.3748/wjg.v26.i19.2416. PubMed PMID: 32476802; PubMed Central PMCID: PMCPMC7243652.

90. Ndrepepa G, Kastrati A. Gamma-glutamyl transferase and cardiovascular disease. Annals of translational medicine. 2016;4(24):481. Epub 2017/02/06. doi: 10.21037/atm.2016.12.27. PubMed PMID: 28149843; PubMed Central PMCID: PMCPMC5233492. 
91. Afolabi PR, Scorletti E, Calder PC, Byrne CD. Factors independently associated with

cardiorespiratory fitness in patients with non-alcoholic fatty liver disease. Liver Int. 2020. Epub 2020/07/25. doi: 10.1111/liv.14618. PubMed PMID: 32706931.

92. Sung KC, Ryu S, Kim BS, Cheong ES, Park DI, Kim BI, et al. gamma-Glutamyl Transferase Is Associated with Mortality Outcomes Independently of Fatty Liver. ClinChem. 2015;61(9):1173-81. doi:

clinchem.2015.240424 [pii];10.1373/clinchem.2015.240424 [doi].

93. Simon TG, Trejo MEP, McClelland R, Bradley R, Blaha MJ, Zeb I, et al. Circulating Interleukin-6 is a biomarker for coronary atherosclerosis in nonalcoholic fatty liver disease: Results from the Multi-Ethnic Study of Atherosclerosis. Int J Cardiol. 2018;259:198-204. Epub 2018/03/27. doi:

10.1016/j.ijcard.2018.01.046. PubMed PMID: 29579601; PubMed Central PMCID: PMCPMC5875712.

94. Moschen AR, Molnar C, Geiger S, Graziadei I, Ebenbichler CF, Weiss H, et al. Anti-inflammatory effects of excessive weight loss: potent suppression of adipose interleukin 6 and tumour necrosis factor alpha expression. Gut. 2010;59(9):1259-64. Epub 2010/07/28. doi: 10.1136/gut.2010.214577. PubMed PMID: 20660075.

95. Mohamed-Ali V, Goodrick S, Rawesh A, Katz DR, Miles JM, Yudkin JS, et al. Subcutaneous adipose tissue releases interleukin-6, but not tumor necrosis factor-alpha, in vivo. J Clin Endocrinol Metab. 1997;82(12):4196-200. Epub 1997/12/17. doi: 10.1210/jcem.82.12.4450. PubMed PMID: 9398739. 96. Wu N, Xu B, Xiang Y, Wu L, Zhang Y, Ma X, et al. Association of inflammatory factors with occurrence and recurrence of atrial fibrillation: a meta-analysis. Int J Cardiol. 2013;169(1):62-72. Epub 2013/10/08. doi: 10.1016/j.ijcard.2013.08.078. PubMed PMID: 24095158.

97. Ridker PM, Everett BM, Thuren T, MacFadyen JG, Chang WH, Ballantyne C, et al. Antiinflammatory Therapy with Canakinumab for Atherosclerotic Disease. N Engl J Med. 2017;377(12):1119-31. Epub 2017/08/29. doi: 10.1056/NEJMoa1707914. PubMed PMID: 28845751.

98. Loomba R, Seguritan V, Li W, Long T, Klitgord N, Bhatt A, et al. Gut Microbiome-Based Metagenomic Signature for Non-invasive Detection of Advanced Fibrosis in Human Nonalcoholic Fatty Liver Disease. Cell Metab. 2017;25(5):1054-62.e5. Epub 2017/05/04. doi: 10.1016/j.cmet.2017.04.001. PubMed PMID: 28467925; PubMed Central PMCID: PMCPMC5502730.

99. Chopyk DM, Grakoui A. Contribution of the Intestinal Microbiome and Gut Barrier to Hepatic Disorders. Gastroenterology. 2020. Epub 2020/06/23. doi: 10.1053/j.gastro.2020.04.077. PubMed PMID: 32569766.

100. Cani PD, Amar J, Iglesias MA, Poggi M, Knauf C, Bastelica D, et al. Metabolic endotoxemia initiates obesity and insulin resistance. Diabetes. 2007;56(7):1761-72. Epub 2007/04/26. doi: 10.2337/db06-1491. PubMed PMID: 17456850.

101. Zhao Y, Wang Z. Impact of trimethylamine N-oxide (TMAO) metaorganismal pathway on cardiovascular disease. Journal of laboratory and precision medicine. 2020;5. Epub 2020/06/27. doi: 10.21037/jlpm.2020.01.01. PubMed PMID: 32587943; PubMed Central PMCID: PMCPMC7316184. 102. Zhuang R, Ge X, Han L, Yu P, Gong X, Meng Q, et al. Gut microbe-generated metabolite trimethylamine $\mathrm{N}$-oxide and the risk of diabetes: A systematic review and dose-response meta-analysis. Obesity reviews : an official journal of the International Association for the Study of Obesity. 2019;20(6):883-94. Epub 2019/03/15. doi: 10.1111/obr.12843. PubMed PMID: 30868721.

103. Tan X, Liu Y, Long J, Chen S, Liao G, Wu S, et al. Trimethylamine N-Oxide Aggravates Liver Steatosis through Modulation of Bile Acid Metabolism and Inhibition of Farnesoid X Receptor Signaling in Nonalcoholic Fatty Liver Disease. Mol Nutr Food Res. 2019;63(17):e1900257. Epub 2019/05/17. doi: 10.1002/mnfr.201900257. PubMed PMID: 31095863.

104. Zeisel SH, Warrier M. Trimethylamine N-Oxide, the Microbiome, and Heart and Kidney Disease. Annual review of nutrition. 2017;37:157-81. Epub 2017/07/19. doi: 10.1146/annurev-nutr-071816-064732. PubMed PMID: 28715991.

105. Janeiro MH, Ramírez MJ, Milagro FI, Martínez JA, Solas M. Implication of Trimethylamine N-Oxide (TMAO) in Disease: Potential Biomarker or New Therapeutic Target. Nutrients. 2018;10(10). Epub 2018/10/03. doi: 10.3390/nu10101398. PubMed PMID: 30275434; PubMed Central PMCID: PMCPMC6213249.

106. Yang K, Xu X, Nie L, Xiao T, Guan X, He T, et al. Indoxyl sulfate induces oxidative stress and hypertrophy in cardiomyocytes by inhibiting the AMPK/UCP2 signaling pathway. Toxicology letters. 2015;234(2):110-9. Epub 2015/02/24. doi: 10.1016/j.toxlet.2015.01.021. PubMed PMID: 25703824. 
107. Wang Z, Zhao Y. Gut microbiota derived metabolites in cardiovascular health and disease. Protein \& cell. 2018;9(5):416-31. Epub 2018/05/05. doi: 10.1007/s13238-018-0549-0. PubMed PMID: 29725935; PubMed Central PMCID: PMCPMC5960473.

108. Scorletti E, Afolabi PR, Miles EA, Smith DE, Almehmadi A, Alshathry A, et al. Synbiotics Alter Fecal Microbiomes, But Not Liver Fat or Fibrosis, in a Randomized Trial of Patients With Nonalcoholic Fatty Liver Disease. Gastroenterology. 2020;158(6):1597-610.e7. Epub 2020/01/29. doi: 10.1053/j.gastro.2020.01.031. PubMed PMID: 31987796.

109. Arnett DK, Blumenthal RS, Albert MA, Buroker AB, Goldberger ZD, Hahn EJ, et al. 2019 ACC/AHA Guideline on the Primary Prevention of Cardiovascular Disease: Executive Summary: A Report of the American College of Cardiology/American Heart Association Task Force on Clinical Practice Guidelines. J Am Coll Cardiol. 2019;74(10):1376-414. Epub 2019/03/22. doi: 10.1016/j.jacc.2019.03.009. PubMed PMID: 30894319.

110. Wilson PW, D'Agostino RB, Levy D, Belanger AM, Silbershatz H, Kannel WB. Prediction of coronary heart disease using risk factor categories. Circulation. 1998;97(18):1837-47. Epub 1998/05/29. doi: 10.1161/01.cir.97.18.1837. PubMed PMID: 9603539.

111. Treeprasertsuk S, Leverage S, Adams LA, Lindor KD, St Sauver J, Angulo P. The Framingham risk score and heart disease in nonalcoholic fatty liver disease. Liver Int. 2012;32(6):945-50. Epub 2012/02/04. doi: 10.1111/j.1478-3231.2011.02753.x. PubMed PMID: 22299674; PubMed Central PMCID: PMCPMC3348257.

112. Goff DC, Jr., Lloyd-Jones DM, Bennett G, Coady S, D'Agostino RB, Gibbons R, et al. 2013 ACC/AHA guideline on the assessment of cardiovascular risk: a report of the American College of Cardiology/American Heart Association Task Force on Practice Guidelines. Circulation. 2014;129(25 Suppl 2):S49-73. Epub 2013/11/14. doi: 10.1161/01.cir.0000437741.48606.98. PubMed PMID: 24222018.

113. Meyersohn NM MT, Corey KE, Bittner DO, Staziaki PV, Szilveszter B, Hallett T, Lu MT, Puchner SB, Simon TG, Foldyna B, Voora D, Ginsburg GS, Douglas BS, Hoffmann U, Ferencik M. Association of Hepatic Steatosis with Major Adverse Cardiovascular Events, Independent of Coronary Artery Disease. Clinical Gastroenterology and Hepatology. 2020. doi: https://doi.org/10.1016/j.cgh.2020.07.030.

114. Cosentino F, Grant PJ, Aboyans V, Bailey CJ, Ceriello A, Delgado V, et al. 2019 ESC Guidelines on diabetes, pre-diabetes, and cardiovascular diseases developed in collaboration with the EASD. Eur Heart J. 2020;41(2):255-323. Epub 2019/09/10. doi: 10.1093/eurheartj/ehz486. PubMed PMID: 31497854.

115. Keating SE, Hackett DA, George J, Johnson NA. Exercise and non-alcoholic fatty liver disease: a systematic review and meta-analysis. J Hepatol. 2012;57(1):157-66. Epub 2012/03/15. doi:

10.1016/j.jhep.2012.02.023. PubMed PMID: 22414768.

116. Estruch R, Ros E, Salas-Salvado J, Covas MI, Corella D, Aros F, et al. Primary Prevention of Cardiovascular Disease with a Mediterranean Diet Supplemented with Extra-Virgin Olive Oil or Nuts. N Engl J Med. 2018;378(25):e34. Epub 2018/06/14. doi: 10.1056/NEJMoa1800389. PubMed PMID: 29897866.

117. Ryan MC, Itsiopoulos C, Thodis T, Ward G, Trost N, Hofferberth S, et al. The Mediterranean diet improves hepatic steatosis and insulin sensitivity in individuals with non-alcoholic fatty liver disease. J Hepatol. 2013;59(1):138-43. Epub 2013/03/15. doi: 10.1016/j.jhep.2013.02.012. PubMed PMID: 23485520. 118. Ma J, Hennein R, Liu C, Long MT, Hoffmann U, Jacques PF, et al. Improved Diet Quality Associates With Reduction in Liver Fat, Particularly in Individuals With High Genetic Risk Scores for Nonalcoholic Fatty Liver Disease. Gastroenterology. 2018;155(1):107-17. Epub 2018/04/01. doi: 10.1053/j.gastro.2018.03.038. PubMed PMID: 29604292; PubMed Central PMCID: PMCPMC6035111.

119. Collaborators GBDT. Smoking prevalence and attributable disease burden in 195 countries and territories, 1990-2015: a systematic analysis from the Global Burden of Disease Study 2015. Lancet. 2017;389(10082):1885-906. Epub 2017/04/10. doi: 10.1016/S0140-6736(17)30819-X. PubMed PMID: 28390697; PubMed Central PMCID: PMCPMC5439023.

120. Angulo P, Kleiner DE, Dam-Larsen S, Adams LA, Bjornsson ES, Charatcharoenwitthaya $P$, et al. Liver Fibrosis, but No Other Histologic Features, Is Associated With Long-term Outcomes of Patients With Nonalcoholic Fatty Liver Disease. Gastroenterology. 2015;149(2):389-97 e10. Epub 2015/05/04. doi: 10.1053/j.gastro.2015.04.043. PubMed PMID: 25935633; PubMed Central PMCID: PMCPMC4516664. 121. Simon TG, Henson J, Osganian S, Masia R, Chan AT, Chung RT, et al. Daily Aspirin Use Associated With Reduced Risk For Fibrosis Progression In Patients With Nonalcoholic Fatty Liver Disease. Clin Gastroenterol Hepatol. 2019;17(13):2776-84 e4. Epub 2019/05/12. doi: 10.1016/j.cgh.2019.04.061. PubMed PMID: 31077838; PubMed Central PMCID: PMCPMC6842070. 
122. Karmali KN, Lloyd-Jones DM, Berendsen MA, Goff DC, Jr., Sanghavi DM, Brown NC, et al. Drugs for Primary Prevention of Atherosclerotic Cardiovascular Disease: An Overview of Systematic Reviews. JAMA Cardiol. 2016;1(3):341-9. Epub 2016/07/22. doi: 10.1001/jamacardio.2016.0218. PubMed PMID: 27438118; PubMed Central PMCID: PMCPMC5053397.

123. Blais $P$, Lin M, Kramer JR, El-Serag HB, Kanwal F. Statins Are Underutilized in Patients with Nonalcoholic Fatty Liver Disease and Dyslipidemia. Dig Dis Sci. 2016;61(6):1714-20. Epub 2015/12/29. doi: 10.1007/s10620-015-4000-6. PubMed PMID: 26707137.

124. Singh S, Singh PP, Singh AG, Murad MH, Sanchez W. Statins are associated with a reduced risk of hepatocellular cancer: a systematic review and meta-analysis. Gastroenterology. 2013;144(2):323-32. Epub 2012/10/16. doi: 10.1053/j.gastro.2012.10.005. PubMed PMID: 23063971.

125. Marso SP, Daniels GH, Brown-Frandsen K, Kristensen P, Mann JF, Nauck MA, et al. Liraglutide and Cardiovascular Outcomes in Type 2 Diabetes. N Engl J Med. 2016;375(4):311-22. Epub 2016/06/14. doi: 10.1056/NEJMoa1603827. PubMed PMID: 27295427; PubMed Central PMCID: PMCPMC4985288.

126. Marso SP, Bain SC, Consoli A, Eliaschewitz FG, Jodar E, Leiter LA, et al. Semaglutide and Cardiovascular Outcomes in Patients with Type 2 Diabetes. N Engl J Med. 2016;375(19):1834-44. Epub 2016/09/17. doi: 10.1056/NEJMoa1607141. PubMed PMID: 27633186.

127. Gerstein HC, Colhoun HM, Dagenais GR, Diaz R, Lakshmanan M, Pais P, et al. Dulaglutide and cardiovascular outcomes in type 2 diabetes (REWIND): a double-blind, randomised placebo-controlled trial. Lancet. 2019;394(10193):121-30. Epub 2019/06/14. doi: 10.1016/S0140-6736(19)31149-3. PubMed PMID: 31189511.

128. Pfeffer MA, Claggett B, Diaz R, Dickstein K, Gerstein HC, Kober LV, et al. Lixisenatide in Patients with Type 2 Diabetes and Acute Coronary Syndrome. N Engl J Med. 2015;373(23):2247-57. Epub 2015/12/03. doi: 10.1056/NEJMoa1509225. PubMed PMID: 26630143.

129. Holman RR, Bethel MA, Mentz RJ, Thompson VP, Lokhnygina Y, Buse JB, et al. Effects of OnceWeekly Exenatide on Cardiovascular Outcomes in Type 2 Diabetes. N Engl J Med. 2017;377(13):1228-39. Epub 2017/09/15. doi: 10.1056/NEJMoa1612917. PubMed PMID: 28910237.

130. Monami M, Nreu B, Scatena A, Giannini S, Andreozzi F, Sesti G, et al. Glucagon-like peptide-1 receptor agonists and atrial fibrillation: a systematic review and meta-analysis of randomised controlled trials. J Endocrinol Invest. 2017;40(11):1251-8. Epub 2017/05/31. doi: 10.1007/s40618-017-0698-7. PubMed PMID: 28569363.

131. Ang R, Mastitskaya S, Hosford PS, Basalay M, Specterman M, Aziz Q, et al. Modulation of Cardiac Ventricular Excitability by GLP-1 (Glucagon-Like Peptide-1). Circ Arrhythm Electrophysiol.

2018;11(10):e006740. doi: 10.1161/CIRCEP.118.006740. PubMed PMID: 30354404; PubMed Central PMCID: PMCPMC6553567.

132. Armstrong MJ, Gaunt P, Aithal GP, Barton D, Hull D, Parker R, et al. Liraglutide safety and efficacy in patients with non-alcoholic steatohepatitis (LEAN): a multicentre, double-blind, randomised, placebocontrolled phase 2 study. Lancet. 2016;387(10019):679-90. Epub 2015/11/27. doi: 10.1016/S01406736(15)00803-X. PubMed PMID: 26608256.

133. Zinman B, Wanner C, Lachin JM, Fitchett D, Bluhmki E, Hantel S, et al. Empagliflozin, Cardiovascular Outcomes, and Mortality in Type 2 Diabetes. N Engl J Med. 2015;373(22):2117-28. Epub 2015/09/18. doi: 10.1056/NEJMoa1504720. PubMed PMID: 26378978.

134. Neal B, Perkovic V, Matthews DR. Canagliflozin and Cardiovascular and Renal Events in Type 2 Diabetes. N Engl J Med. 2017;377(21):2099. Epub 2017/11/23. doi: 10.1056/NEJMc1712572. PubMed PMID: 29166232.

135. Yurista SR, Silljé HHW, Rienstra M, de Boer RA, Westenbrink BD. Sodium-glucose co-transporter 2 inhibition as a mitochondrial therapy for atrial fibrillation in patients with diabetes? Cardiovasc Diabetol. 2020;19(1):5. Epub 2020/01/07. doi: 10.1186/s12933-019-0984-0. PubMed PMID: 31910841; PubMed Central PMCID: PMCPMC6945755.

136. Shao Q, Meng L, Lee S, Tse G, Gong M, Zhang Z, et al. Empagliflozin, a sodium glucose cotransporter-2 inhibitor, alleviates atrial remodeling and improves mitochondrial function in high-fat diet/streptozotocin-induced diabetic rats. Cardiovasc Diabetol. 2019;18(1):165. Epub 2019/11/28. doi: 10.1186/s12933-019-0964-4. PubMed PMID: 31779619; PubMed Central PMCID: PMCPMC6882319. 137. Kuchay MS, Krishan S, Mishra SK, Farooqui KJ, Singh MK, Wasir JS, et al. Effect of Empagliflozin on Liver Fat in Patients With Type 2 Diabetes and Nonalcoholic Fatty Liver Disease: A Randomized Controlled Trial (E-LIFT Trial). Diabetes Care. 2018;41(8):1801-8. Epub 2018/06/14. doi: 10.2337/dc18-0165. PubMed PMID: 29895557. 
138. Lai LL, Vethakkan SR, Nik Mustapha NR, Mahadeva S, Chan WK. Empagliflozin for the Treatment of Nonalcoholic Steatohepatitis in Patients with Type 2 Diabetes Mellitus. Dig Dis Sci. 2020;65(2):623-31. Epub 2019/01/25. doi: 10.1007/s10620-019-5477-1. PubMed PMID: 30684076.

139. Kahl S, Gancheva S, Straßburger K, Herder C, Machann J, Katsuyama H, et al. Empagliflozin Effectively Lowers Liver Fat Content in Well-Controlled Type 2 Diabetes: A Randomized, Double-Blind, Phase 4, Placebo-Controlled Trial. Diabetes Care. 2020;43(2):298-305. Epub 2019/09/20. doi:

10.2337/dc19-0641. PubMed PMID: 31540903.

140. Taheri H, Malek M, Ismail-Beigi F, Zamani F, Sohrabi M, Reza Babaei M, et al. Effect of Empagliflozin on Liver Steatosis and Fibrosis in Patients With Non-Alcoholic Fatty Liver Disease Without Diabetes: A Randomized, Double-Blind, Placebo-Controlled Trial. Adv Ther. 2020;37(11):4697-708. Epub 2020/09/25. doi: 10.1007/s12325-020-01498-5. PubMed PMID: 32975679.

141. Cusi K, Bril F, Barb D, Polidori D, Sha S, Ghosh A, et al. Effect of canagliflozin treatment on hepatic triglyceride content and glucose metabolism in patients with type 2 diabetes. Diabetes Obes Metab. 2019;21(4):812-21. Epub 2018/11/18. doi: 10.1111/dom.13584. PubMed PMID: 30447037.

142. Belfort R, Harrison SA, Brown K, Darland C, Finch J, Hardies J, et al. A placebo-controlled trial of pioglitazone in subjects with nonalcoholic steatohepatitis. N Engl J Med. 2006;355(22):2297-307. Epub 2006/12/01. doi: 10.1056/NEJMoa060326. PubMed PMID: 17135584.

143. Aithal GP, Thomas JA, Kaye PV, Lawson A, Ryder SD, Spendlove I, et al. Randomized, placebocontrolled trial of pioglitazone in nondiabetic subjects with nonalcoholic steatohepatitis. Gastroenterology. 2008;135(4):1176-84. Epub 2008/08/23. doi: 10.1053/j.gastro.2008.06.047. PubMed PMID: 18718471.

144. Sanyal AJ, Chalasani N, Kowdley KV, McCullough A, Diehl AM, Bass NM, et al. Pioglitazone, vitamin E, or placebo for nonalcoholic steatohepatitis. N Engl J Med. 2010;362(18):1675-85. Epub 2010/04/30. doi: 10.1056/NEJMoa0907929. PubMed PMID: 20427778; PubMed Central PMCID: PMCPMC2928471.

145. Mantovani A, Byrne CD, Scorletti E, Mantzoros CS, Targher G. Efficacy and safety of antihyperglycaemic drugs in patients with non-alcoholic fatty liver disease with or without diabetes: An updated systematic review of randomized controlled trials. Diabetes Metab. 2020. Epub 2020/01/11. doi: 10.1016/j.diabet.2019.12.007. PubMed PMID: 31923578.

146. Cusi K, Orsak B, Bril F, Lomonaco R, Hecht J, Ortiz-Lopez C, et al. Long-Term Pioglitazone Treatment for Patients With Nonalcoholic Steatohepatitis and Prediabetes or Type 2 Diabetes Mellitus: A Randomized Trial. Ann Intern Med. 2016;165(5):305-15. Epub 2016/06/21. doi: 10.7326/M15-1774. PubMed PMID: 27322798.

147. Musso G, Cassader M, Paschetta E, Gambino R. Thiazolidinediones and Advanced Liver Fibrosis in Nonalcoholic Steatohepatitis: A Meta-analysis. JAMA Intern Med. 2017;177(5):633-40. Epub 2017/02/28. doi: 10.1001/jamainternmed.2016.9607. PubMed PMID: 28241279; PubMed Central PMCID: PMCPMC5470366.

148. Chalasani N, Younossi Z, Lavine JE, Charlton M, Cusi K, Rinella M, et al. The diagnosis and management of nonalcoholic fatty liver disease: Practice guidance from the American Association for the Study of Liver Diseases. Hepatology. 2018;67(1):328-57. Epub 2017/07/18. doi: 10.1002/hep.29367. PubMed PMID: 28714183.

149. European Association for the Study of the L, European Association for the Study of D, European Association for the Study of O. EASL-EASD-EASO Clinical Practice Guidelines for the management of nonalcoholic fatty liver disease. J Hepatol. 2016;64(6):1388-402. Epub 2016/04/12. doi:

10.1016/j.jhep.2015.11.004. PubMed PMID: 27062661.

150. Dormandy JA, Charbonnel B, Eckland DJ, Erdmann E, Massi-Benedetti M, Moules IK, et al. Secondary prevention of macrovascular events in patients with type 2 diabetes in the PROactive Study (PROspective pioglitAzone Clinical Trial In macroVascular Events): a randomised controlled trial. Lancet. 2005;366(9493):1279-89. Epub 2005/10/11. doi: 10.1016/S0140-6736(05)67528-9. PubMed PMID: 16214598.

151. Kernan WN, Viscoli CM, Furie KL, Young LH, Inzucchi SE, Gorman M, et al. Pioglitazone after Ischemic Stroke or Transient Ischemic Attack. N Engl J Med. 2016;374(14):1321-31. Epub 2016/02/18. doi: 10.1056/NEJMoa1506930. PubMed PMID: 26886418; PubMed Central PMCID: PMCPMC4887756.

152. Spence JD, Viscoli CM, Inzucchi SE, Dearborn-Tomazos J, Ford GA, Gorman M, et al. Pioglitazone Therapy in Patients With Stroke and Prediabetes: A Post Hoc Analysis of the IRIS Randomized Clinical Trial. JAMA Neurol. 2019;76(5):526-35. Epub 2019/02/09. doi: 10.1001/jamaneurol.2019.0079. PubMed PMID: 30734043; PubMed Central PMCID: PMCPMC6515584. 
153. Corey KE, Vuppalanchi R, Wilson LA, Cummings OW, Chalasani N, Nash CRN. NASH resolution is associated with improvements in HDL and triglyceride levels but not improvement in LDL or non-HDL-C levels. Aliment Pharmacol Ther. 2015;41(3):301-9. Epub 2014/11/29. doi: 10.1111/apt.13035. PubMed PMID: 25429853; PubMed Central PMCID: PMCPMC4424085.

154. Corey KE, Wilson LA, Altinbas A, Yates KP, Kleiner DE, Chung RT, et al. Relationship between resolution of non-alcoholic steatohepatitis and changes in lipoprotein sub-fractions: a post-hoc analysis of the PIVENS trial. Aliment Pharmacol Ther. 2019;49(9):1205-13. Epub 2019/03/12. doi: 10.1111/apt.15216. PubMed PMID: 30854694; PubMed Central PMCID: PMCPMC6461513.

155. Joseph J, Velasco A, Hage FG, Reyes E. Guidelines in review: Comparison of ESC and ACC/AHA guidelines for the diagnosis and management of patients with stable coronary artery disease. J Nucl Cardiol. 2018;25(2):509-15. Epub 2017/09/09. doi: 10.1007/s12350-017-1055-0. PubMed PMID: 28884447.

156. Connelly MA, Velez Rivera J, Guyton JR, Siddiqui MS, Sanyal AJ. Review article: the impact of liverdirected therapies on the atherogenic risk profile in non-alcoholic steatohepatitis. Aliment Pharmacol Ther. 2020;52(4):619-36. Epub 2020/07/08. doi: 10.1111/apt.15935. PubMed PMID: 32638417; PubMed Central PMCID: PMCPMC7497003.

\section{FIGURE LEGENDS}

Figure 1. NAFLD-related effects on coronary arteries and other structures of the heart. NAFLD not only promotes accelerated coronary atherosclerosis, but also increases the risk of cardiomyopathy, cardiac valvular calcification, arrhythmias (mostly permanent atrial fibrillation) and some cardiac conduction defects. Abbreviations: NAFL, non-alcoholic fatty liver; NASH, non-alcoholic steatohepatitis

Figure 2. Inter-connections among NAFLD, type 2 diabetes, metabolic syndrome and risk of cardiovascular disease and cardiac/arrhythmic complications. The figure illustrates schematically in Panel A, the "vicious spiral" of worsening disease that occurs when NAFLD co-exists with type 2 diabetes (T2DM). Current evidence suggests that with the presence of NAFLD, both steatosis and liver fibrosis are associated with an increase in risk of incident cardiovascular disease (CVD). Presently it is uncertain whether any specific histological feature of NAFLD contributes a differentially greater risk of CVD. The presence of either NAFLD or T2DM increases the risk of, and development and progression, of the other disease. For example, liver 
fat increases the risk of incident T2DM and with further progression of liver disease to liver fibrosis, the risk of T2DM is further increased. The coexistence of T2DM with NAFLD often makes it difficult to achieve good glycemic control, due to marked hepatic and peripheral insulin resistance. T2DM also increases progression of liver disease, with increased risk of NASH, advanced fibrosis, cirrhosis or hepatocellular carcinoma. When NAFLD and T2DM coexist, both conditions independently increase the risk of CVD and cardiac diseases. Panel B shows some of the key potential CVD and cardiac risk factors in patients with NAFLD.

Figure 3. Putative pathophysiological links between NAFLD, expanded adipose tissue and intestinal dysbiosis with the development of atherogenic dyslipidaemia. This figure illustrates schematically the development of atherogenic dyslipidaemia in NAFLD and its relationship with features of the metabolic syndrome. The figure also highlights the role of lipopolysaccharide (LPS), chylomicrons and adipose-derived factors, such as long-chain fatty acids (LCFAs), inflammatory cytokines and adipokines to influence liver lipid species [e.g., ceramides, di-palmitoyl phosphatidic acid (di-P PA) and diacylglycerols (DAGs)] that may affect liver inflammatory processes, insulin sensitivity and oxidative stress. Some NAFLD-related genetic variants, such as PNPLA3 148M and TM6SF2 167K (see text), may modify the accumulation of the lipid globule and very-low density lipoprotein (VLDL) production and secretion as a key component of the atherogenic lipoprotein profile and the presence of these genotypes may act to differentially modify CVD risk in patients with NAFLD. Two elements of the atherogenic lipoprotein profile [i.e., increased VLDL and decreased high-density lipoprotein (HDL) particles] are also both key components of the clinically diagnosed metabolic syndrome (i.e., increased VLDL particles manifest as increased fasting triglyceride concentrations and decreased HDL-cholesterol concentrations) that often occur with NAFLD and contribute to increased CVD risk.

Figure 4. Possible therapeutic approaches that reduce CVD risk and potentially modify also liver disease. The management of adult individuals with NAFLD to reduce the risk of CVD has yet to be entirely defined. The possible therapeutic approaches are formulated to target the underlying mechanisms that associate NAFLD and CVD. Abbreviations: ACE, angiotensin-converting enzyme; SGLT2, sodiumglucose cotransporter-2; GLP-1 RA, glucagon-like peptide-1 receptor agonist; HF, heart failure; HCC, hepatocellular carcinoma. 
Table 1. Meta-analytic quantification of the excess of CVD risk (markers of subclinical atherosclerosis and major adverse CVD events), arrhythmias (atrial fibrillation) and presence of cardiac dysfunction or aorticvalve sclerosis in individuals with NAFLD.

\begin{tabular}{|c|c|c|c|}
\hline Author, Reference & $\begin{array}{l}\text { Study } \\
\text { characteristics }\end{array}$ & Markers/outcomes & $\begin{array}{l}\text { Random- } \\
\text { effects OR or } \\
\text { HR ( } 95 \% \\
\text { confidence } \\
\text { intervals) } \\
\end{array}$ \\
\hline \multicolumn{4}{|c|}{ Markers of subclinical atherosclerosis } \\
\hline \multirow[t]{4}{*}{ Zhou YY et al. [11] } & \multirow{4}{*}{$\begin{array}{l}26 \text { cross-sectional } \\
\text { studies involving a } \\
\text { total of } 85,395 \\
\text { individuals } \\
\text { ( } 28.9 \% \text { with } \\
\text { NAFLD) }\end{array}$} & $\begin{array}{l}\text { Increased carotid artery } \\
\text { intima-media } \\
\text { thickness/plaques }\end{array}$ & $1.74(1.47-2.06)$ \\
\hline & & $\begin{array}{l}\text { Increased arterial } \\
\text { stiffness }\end{array}$ & 1.56 (1.24-1.96) \\
\hline & & $\begin{array}{l}\text { Coronary artery } \\
\text { calcification }\end{array}$ & $1.40(1.22-1.60)$ \\
\hline & & $\begin{array}{l}\text { Circulatory endothelial } \\
\text { dysfunction }\end{array}$ & $3.73(0.99-14.1)$ \\
\hline \multicolumn{4}{|c|}{ Fatal and non-fatal CVD events ${ }^{\S}$} \\
\hline \multirow[t]{6}{*}{$\begin{array}{l}\text { Targher } \mathrm{G} \text { et al. } \\
\text { [12] }\end{array}$} & \multirow{4}{*}{$\begin{array}{l}16 \text { longitudinal } \\
\text { studies involving a } \\
\text { total of } 34,043 \\
\text { individuals ( } 36.3 \% \\
\text { with NAFLD); } \\
\text { median follow-up of } \\
6.9 \text { years }\end{array}$} & $\begin{array}{l}\text { Any fatal or nonfatal } \\
\text { CVD events, } n=16 \\
\text { studies }\end{array}$ & $1.64(1.26-2.13)$ \\
\hline & & $\begin{array}{l}\text { Fatal CVD events (only), } \\
\mathrm{n}=6 \text { studies }\end{array}$ & $1.31(0.87-1.97)$ \\
\hline & & $\begin{array}{l}\text { Fatal and nonfatal CVD } \\
\text { events (combined } \\
\text { endpoint), } n=5 \text { studies }\end{array}$ & $1.63(1.06-2.48)$ \\
\hline & & $\begin{array}{l}\text { Nonfatal CVD events } \\
\text { (only), } n=5 \text { studies }\end{array}$ & $2.52(1.52-4.18)$ \\
\hline & \multirow{2}{*}{$\begin{array}{l}\text { Subgroup analyses } \\
\text { in patients with } \\
\text { "more severe" } \\
\text { NAFLD* (n=6 } \\
\text { studies) }\end{array}$} & $\begin{array}{l}\text { Fatal CVD events (only), } \\
n=3 \text { studies }\end{array}$ & $3.28(2.26-4.77)$ \\
\hline & & $\begin{array}{l}\text { Fatal and nonfatal CVD } \\
\text { events (combined } \\
\text { endpoint), } n=3 \text { studies }\end{array}$ & $1.94(1.17-3.21)$ \\
\hline \multicolumn{4}{|l|}{ Atrial fibrillation } \\
\hline \multirow[t]{2}{*}{$\begin{array}{l}\text { Mantovani A et al. } \\
\text { [22] }\end{array}$} & \multirow{2}{*}{$\begin{array}{l}5 \text { cross-sectional } \\
\text { and } 4 \text { longitudinal } \\
\text { studies involving a } \\
\text { total of } 364,919 \\
\text { individuals ( } 43 \% \\
\text { with NAFLD) }\end{array}$} & $\begin{array}{l}\text { Prevalent atrial } \\
\text { fibrillation, } n=5 \text { studies }\end{array}$ & $\begin{array}{l}2.07(1.38-3.10) \\
5.17(2.05-13.0) \\
\text { for type } 2 \\
\text { diabetics only }\end{array}$ \\
\hline & & $\begin{array}{l}\text { Incident atrial fibrillation, } \\
n=4 \text { studies }\end{array}$ & $\begin{array}{l}1.34(0.92-1.95) \\
4.96(1.42-17.3) \\
\text { for type } 2 \\
\text { diabetics only }\end{array}$ \\
\hline Cai X et al. [23] & $\begin{array}{l}6 \text { longitudinal } \\
\text { studies involving a } \\
\text { total of } 614,673 \\
\text { individuals }(\sim 40 \% \\
\text { with NAFLD); } \\
\text { median follow-up of } \\
10 \text { years }\end{array}$ & $\begin{array}{l}\text { Incident atrial fibrillation, } \\
\mathrm{n}=6 \text { studies }\end{array}$ & $1.19(1.04-1.31)$ \\
\hline \multicolumn{4}{|l|}{ Cardiac dysfunction } \\
\hline $\begin{array}{l}\text { Wijarnpreecha K et } \\
\text { al. [29] }\end{array}$ & $\begin{array}{l}12 \text { cross-sectional } \\
\text { studies involving a } \\
\text { total of } 280,645 \\
\text { individuals }(\sim 30 \% \\
\text { with NAFLD) }\end{array}$ & $\begin{array}{l}\text { Left ventricular diastolic } \\
\text { dysfunction on } \\
\text { echocardiography }\end{array}$ & $2.02(1.47-2.79)$ \\
\hline \multicolumn{4}{|c|}{ Aortic-valve sclerosis } \\
\hline $\begin{array}{l}\text { Di Minno MN et al. } \\
\text { [38] }\end{array}$ & $\begin{array}{l}3 \text { cross-sectional } \\
\text { studies involving a } \\
\text { total of } 2,639 \\
\text { individuals ( } 44.4 \% \\
\text { with NAFLD) }\end{array}$ & $\begin{array}{l}\text { Aortic-valve sclerosis on } \\
\text { echocardiography }\end{array}$ & $2.28(1.21-4.28)$ \\
\hline
\end{tabular}


$\S$ Fatal and/or non-fatal CVD events were defined as presence of cardiovascular death, non-fatal CVD events (i.e., acute myocardial infarction, angina, ischemic stroke or coronary revascularization procedures), or both.

* Defined by the presence of either NAFLD on imaging techniques plus either elevated serum gamma-glutamyltransferase concentrations or high NAFLD fibrosis score or high F-18 fluoro-deoxyglucose uptake on positron emission tomography, or by increasing liver fibrosis stage on histology. 